Title: Design, Fabrication, and In Vitro Testing of Novel Three-Dimensionally Printed Tympanic Membrane Grafts

Authors: Elliott D. Kozin ${ }^{1,2,3}$, Nicole L. Black ${ }^{4,5}$, Jeffrey T. Cheng ${ }^{1,2,3}$, Max J. Cotler ${ }^{4,5}$, Michael J. McKenna ${ }^{1,2,3}$, Daniel J. Lee ${ }^{1,2,3}$, Jennifer A. Lewis ${ }^{4,5}$, John J. Rosowski ${ }^{1,2,3}$, Aaron K. Remenschneider ${ }^{1,2,3,{ }^{*}}$

\footnotetext{
Affiliation:

${ }^{1}$ Department Otolaryngology, Massachusetts Eye and Ear Infirmary, Boston, Massachusetts, United States of America

${ }^{2}$ Eaton Peabody Laboratories, Massachusetts Eye and Ear Infirmary, Boston, Massachusetts, United States of America

${ }^{3}$ Department of Otology and Laryngology, Harvard Medical School, Boston, Massachusetts, United States of America

${ }^{4}$ Harvard John A. Paulson School of Engineering and Applied Sciences, Harvard University, Cambridge, Massachusetts, United States of America

${ }^{5}$ Wyss Institute for Biologically Inspired Engineering, Harvard University, Cambridge, Massachusetts, United States of America
}

Conflict of Interest: None

Financial Disclosure: None

Keywords: tympanic membrane, 3D printing, holography, tympanoplasty, biomimetic, tissue engineering

Formatted for: Hearing Research

Word Count: 6485

Corresponding Author:

Aaron_Remenschneider@meei.harvard.edu (AKR) 


\section{Commonly Used Abbreviations}

Chronic otitis media (COM)

Digital opto-electronic holography (DOEH)

Direct ink writing (DIW)

Dynamic mechanical analysis (DMA)

Flex-polyactic acid (PLA)

Laser Doppler vibrometry (LDV)

Polycaprolactone (PCL)

Polydimethylsiloxane (PDMS)

Three-dimensional (3D)

Tympanic membrane (TM) 


\begin{abstract}
The tympanic membrane (TM) is an exquisite structure that captures and transmits sound from the environment to the ossicular chain of the middle ear. The creation of TM grafts by multi-material three-dimensional (3D) printing may overcome limitations of current graft materials, e.g. temporalis muscle fascia, used for surgical reconstruction of the TM. TM graft scaffolds with either 8 or 16 circumferential and radial filament arrangements were fabricated by 3D printing of polydimethylsiloxane (PDMS), flex-polyactic acid (PLA) and polycaprolactone (PCL) materials followed by uniform infilling with a fibrin-collagen composite hydrogel. Digital opto-electronic holography (DOEH) and laser Doppler vibrometry (LDV) were used to measure acoustic properties including surface motions and velocity of TM grafts in response to sound. Mechanical properties were determined using dynamic mechanical analysis (DMA). Results were compared to fresh cadaveric human TMs and cadaveric temporalis fascia. Similar to the human TM, TM grafts exhibit simple surface motion patterns at lower frequencies (400 $\mathrm{Hz})$, with a limited number of displacement maxima. At higher frequencies $(>1000 \mathrm{~Hz})$, their displacement patterns are highly organized with multiple areas of maximal displacement separated by regions of minimal displacement. By contrast, temporalis fascia exhibited asymmetric and less regular holographic patterns. Velocity across frequency sweeps $(0.2-10 \mathrm{kHz})$ measured by LDV demonstrated consistent results for 3D printed grafts, while velocity for human fascia varied greatly between specimens. TM composite grafts of different scaffold print materials and varied filament count ( 8 or 16) displayed minimal, but measurable differences in DOEH and LDV at tested frequencies. TM graft mechanical load increased with higher filament count and is resilient over time,
\end{abstract}


which differs from temporalis fascia, which loses over $70 \%$ of its load bearing properties during mechanical testing. This study demonstrates the design, fabrication and preliminary in vitro acoustic and mechanical evaluation of 3D printed TM grafts. Data illustrate the feasibility of creating TM grafts with acoustic properties that reflect sound induced motion patterns of the human TM; furthermore, 3D printed grafts have mechanical properties that demonstrate increased resistance to deformation compared to temporalis fascia.

This work was presented at MEMRO 2015 in Aalborg Denmark. 


\section{Introduction}

The tympanic membrane (TM) captures and directs sound from the environment to the ossicular chain of the middle ear, enabling transformation of sound pressure waves to mechanical motion. Mechanical motion is subsequently transmitted by the ossicles to the inner ear, where movement of perilymph results in stimulation of the hair cells, which convert mechanical energy into neuronal impulses. The dynamic functional properties of the TM are contained in a multi-layered structure that is less than $100 \mu \mathrm{m}$ thick (Kuypers et al., 2006). A host of structural features, including collagen fiber arrangement and trilayer design, enables the conversion of environmental sound to mechanical motion (O'Connor et al., 2008). These unique structural features work in concert to enable the effective transmission of sound across a wide range of frequencies (humans: $20 \mathrm{~Hz}$ to $20,000 \mathrm{~Hz}$ ) and result in complex movements that vary in magnitude from picometers to microns (Decraemer et al., 1991).

Damage to the TM, such as from chronic otitis media (COM) or traumatic perforation, results in hearing loss due to ineffective sound transmission (Strens et al., 2012). Suppurative COM affects over 30 million individuals worldwide each year, leading to a significant health care burden (Monasta et al., 2012). The most common long-term complication in patients with COM is persistent TM perforation and conductive hearing loss. Tympanoplasty is the surgical repair of the TM, a procedure performed tens of thousands of times each year in the United States (Rubin, 1982). Successful tympanoplasty re-establishes efficient sound transmission from the environment to the ossicular chain, while also recreating a robust barrier between the ear canal and middle ear. 
Historically, cadaveric TMs (House et al., 1966; Marquet, 1971), bovine pericardium (Pfaltz and Griesemer, 1985), and simple synthetic matrices (Kohn et al., 1984; Levin et al., 2009) have been used as TM grafts with moderate success in tympanoplasty. However, due to infectious risk from cadaveric tissue and concerns over graft uptake in synthetic matrices, the most common materials used today are autologous temporalis fascia, perichondrium and cartilage, which can be harvested from the patient at the time of surgery (Cabra and Monux, 2010; Eviatar, 1978; Lyons et al., 2015). Further, studies have indicated the ability of fascia to remodel, thin and become translucent following placement (Szabo, 2006).

Despite its widespread use and proven efficacy to reconstruct the TM and restore hearing, fascia has inherent limitations as a graft material. Fascia may contain intrinsic defects rendering it susceptible to ongoing COM (Boedts et al., 1990; Hiraide et al., 1980). The small irregularities within temporalis fascia may not be perceptible at the time of surgery, leading to unpredictable outcomes. Like all graft materials, fascia is also susceptible to continued middle-ear pressure differences that can result in retraction or re-perforation of the TM. Recent studies demonstrate that perforations persist in greater than $15 \%$ of pediatric patients following primary tympanoplasty (Hardman et al., 2015) and revision surgery rates for patients with COM nearly double that rate to $28 \%$ (Kaylie et al., 2006). Furthermore, revision procedures often leave the surgeon without adequate graft materials for TM reconstruction, given limited quantities of autologous materials. Collectively, refinements in graft materials used in tympanoplasty may improve patient outcomes and decrease surgery-related morbidity in selected cases. 
Recent advances in multi-material three-dimensional (3D) printing enable fabrication of complex microscale architectures with controlled composition and structure (Gratson et al., 2004; Mironov et al., 2003; Murphy and Atala, 2014; Sun et al., 2012). Of specific relevance is direct ink writing (DIW), an extrusion-based printing method that enables a broad range of viscoelastic materials to be patterned under ambient conditions (Barry et al., 2009; Gratson et al., 2004; Hanson Shepherd et al., 2011; Lewis, 2006). Here, we harness advanced 3D printing techniques in an attempt to design and fabricate a biomimetic TM graft with the goal of reproducing specific structural features of the human TM.

\section{Methods}

\subsubsection{Printed 'biomimetic' tympanic membrane design}

To facilitate the design of a biomimetic TM graft, scanning and transmission electron microscopy images of the human TM are used as a guide (Knutsson et al., 2009; Lim, 1968; Lim, 1995; Shimada and Lim, 1971). Observations of radial, circumferential, and parabolic fibers suggest an intrinsic fibrous structure of the TM and its attachments to the fibrous/bony annulus and malleus. Radial and circumferential fibrous arrangements of two separate filament counts $(8$ circumferential $[C] \times 8$ radial $[R]$ or $16 \mathrm{C} \times 16 \mathrm{R}$ ) were chosen for evaluation. Given that graft diameters used for total TM replacement standardly measure $15 \mathrm{~mm}$ in diameter (Sheehy and Anderson, 1980), we produced an ultra-structural TM scaffold design of identical size. The graft was supported by an additional $10 \mathrm{~mm}$ border region for mounting during in-vitro testing for a 
final diameter of $25 \mathrm{~mm}$. Grafts are designed within the AeroBasic software package (Pittsburgh, PA, US).

\subsection{Materials System}

Three different synthetic polymers were used to create 3D TM graft "scaffolds": polydimethylsiloxane (SE 1700 PDMS, Dow Chemical, MA, USA), flexible polylactic acid (PLA, fbrc8, Bartlett, TN, USA), and polycaprolactone (PCL, MW 43,000-50,000, Polysciences Inc, PA, USA). Silicone elastomers (PDMS) are used in a variety of medical implants due to their elastomeric mechanical properties (Calobrace, 2014; Lin et al., 2014). PDMS is non-toxic and is considered to be non-biodegradable(Lukasiak et al., 2005). By contrast, PLA and PCL are biodegradable thermoplastic polymers widely used in biomedical applications and drug delivery (Chu et al., 1995; Dhatta, 1995; Kulkarni et al., 1971; Smith, 1986).

DIW utilizes viscoelastic inks that are extruded through fine deposition nozzles under ambient conditions in filamentary form. SE1700 PDMS is a commercially available silicone that is suitable for printing in its as-received form. PLA and PCL were dissolved in a solvent to produce printable inks under ambient conditions. Specifically, PLA was dissolved at $20 \mathrm{wt} \%$ in hexafluoro-2-propanol, which is a fluorinated alcohol used previously to create bony implants (Uebersax et al., 2013). PCL was dissolved at $50 \mathrm{wt} \%$ in toluene, which is a halogenated Class 2 solvent approved for limited use in pharmaceutical products at a permitted daily exposure of $8.9 \mathrm{mg} /$ day and concentration limit of 890 parts per million (Galow et al., 2002). Residual HFIP and toluene were

removed from the final printed polymer form using rotary evaporation (Hardy et al., 2015). 


\subsection{D Printing of tympanic membranes}

A custom-designed, multi-material 3D printer with an overall build volume of 725 $\mathrm{mm} \times 650 \mathrm{~mm} \times 150 \mathrm{~mm}$ (ABG 10000, Aerotech Inc., Pittsburgh, PA, USA), was used to fabricate TM graft scaffolds (Fig. 1A). It was equipped with four independent, addressable ink reservoirs for DIW of complex 3D architectures at the microscale. Patterned features composed of cylindrical filaments with a minimum diameter of $1 \mu \mathrm{m}$ can be created by this approach (Barry et al., 2009; Hanson Shepherd et al., 2011). TM graft scaffolds composed of PDMS were printed using tapered steel nozzles (100 $\mu \mathrm{m}$ inner diameter; GDP Global, Grand Junction, CO, USA), while those composed of PLA and PCL were printed using straight steel nozzles of similar inner diameter (Nordson EFD, RI, USA), (Fig. 1B-D and 2). The outer 'border region' of the graft, which consists of one ring overlapping the outmost circular filaments of the TM graft scaffold (Fig. 1C) was printed with a $610 \mu \mathrm{m}$ diameter nozzle. The border region (Fig. 2D) stabilizes the TM graft scaffolds and allows handling and mounting for acoustic tests (digital opto-electronic holography and laser Doppler vibrometry).

Printed TM graft scaffolds were cured or dried to achieve their desired solid form. Printed PDMS TM graft scaffolds were placed in an oven (Fisher Scientific, MA, USA) and cured at $100^{\circ} \mathrm{C}$ for $2 \mathrm{~h}$. They were then plasma treated (Femto PCCE plasma cleaner, Diener Electronic, Germany) to render their surface hydrophilic. The printed PLA and PCL scaffolds solidify upon solvent evaporation. Filament widths were determined by optical microscopy coupled with image analysis using ImageJ software (National Institutes of Health, Bethesda, MD, USA) on 10 representative TM scaffolds produced from each 
polymer. Filament thicknesses were measured for these same scaffolds using a micrometer.

\subsection{Infilling Tympanic Membrane Graft Scaffolds}

To create an environment that mimics the natural extracellular matrix of human $\mathrm{TM}$, biodegradable hydrogels known to promote cell ingrowth were used to infill the filamentary skeleton of the TM graft scaffolds (Geckil et al., 2010; Seliktar, 2012). Uniform infill creates a confluent membrane structure for sound transmission and acoustic testing. The TM graft scaffolds were placed on Bytac-coated glass slides (Saint Gobain, PA, USA) to facilitate removal following curing. The slides were placed on top of a hotplate held at $37^{\circ} \mathrm{C}$. The infill matrix was prepared by first creating a solution of $80 \mathrm{mg} / \mathrm{mL}$ fibrinogen (Calbiochem, San Diego, CA, USA) from bovine plasma in Dulbecco's Phosphate-Buffered Saline. This solution was placed on the hotplate and held at $37^{\circ} \mathrm{C}$ for $1 \mathrm{~h}$ to allow for complete solubilization of the protein. Next, a solution of $3 \mathrm{mg} / \mathrm{mL}$ Rat collagen I (Cultrex, Trevigen, Gaithersburg, MD, USA) was neutralized with a buffer containing N-2-hydroxyethylpiperazine-N'-2-ethanesulfonic acid and Dulbecco's Phosphate-Buffered Saline. The two solutions were mixed to obtain a final infill matrix composition of $40 \mathrm{mg} / \mathrm{mL}$ fibrinogen and $1 \mathrm{mg} / \mathrm{mL}$ collagen. From this bulk mixture, 100 $\mu \mathrm{L}$ was pipetted into $2 \mu \mathrm{L}$ of human high activity thrombin enzyme at $1000 \mathrm{U}$ (Calbiochem San Diego, CA, USA). The resultant aliquot was evenly deposited over the top of the TM graft scaffold via a pipette to obtain a uniform infill between printed filaments. The slides were covered to prevent evaporation during the enzymatic conversion process (soluble fibrinogen was converted to fibrin: a non-globular polymerized form, by thrombin) and left 
on the hotplate for $30 \mathrm{~min}$. Final TM composite grafts, consisting of the 3D printed polymer scaffold and infilled solidified biopolymer matrix, were removed from the slide and placed in deinonized water. They were stored at $37^{\circ} \mathrm{C}$ until testing was performed.

\subsection{Human temporalis fascia and tympanic membranes}

The properties of the TM grafts were compared to human temporalis fascia and TMs. Human temporalis fascia was harvested from fresh, unfixed cadaveric specimens by a standard surgical procedure. Specifically, a vertical temporal scalp incision was made with a scalpel and the temporalis fascia was exposed. The fascia was incised and separated from the underlying temporalis muscle. The obtained tissue, approximately $30 \mathrm{~mm} \times 30 \mathrm{~mm}$, was harvested from the superior, thin region of the temporalis fascia. Once harvested, any remaining soft tissue including muscle or fat, was removed. The fascia was then washed in sterile saline, cut to a $25 \mathrm{~mm} \times 25 \mathrm{~mm}$ piece, and mounted for testing using the same techniques as described in 2.6-8.

Human TMs were obtained from fresh cadaveric middle ears. The harvest and preparation of specimens has been previously described (Cheng et al., 2013; Cheng et al., 2010). In brief, the intact temporal bone was extracted shortly after death. The middle ear was opened from the mastoid to observe the ossicular chain, which was left intact. The bony and cartilaginous ear canal lateral to the TM was removed. Testing was performed on the lateral surface of the TM using techniques described in 2.6-7. The institutional Massachusetts Eye and Ear Infirmary Human Studies Committee reviewed and exempted the project (Protocol \#: 785344-1). 
It is important to note that the comparison of 3D printed TM grafts to human cadaveric TMs is limited by inherent differences in middle ear load and conical shape. Data obtained herein is intended to provide a preliminary evaluation of scaffold design, acoustic and mechanical properties with a vision towards future studies using conically shaped TMs with associated ossicular and cochlear loads.

\subsection{Digital Opto-Electronic Holography}

Digital Opto-Electronic Holography (DOEH) interferometer records motion-induced holograms in real-time through two interfering laser beams, providing qualitative and quantitative full-field information on the sound induced motion of a membrane (Cheng et al., 2010; Del Socorro Hernández-Montes et al., 2009). The magnitude and phase angle of displacement of more than 400,000 points on the surface of the membrane can be acquired simultaneously. Briefly, each membrane was held in a customized holder (Fig. S1) placed against a sound coupler integrated into the interferometer camera head and oriented such that the surface of the membrane was perpendicular to the object beam of the laser. When mounted in the holder, the border region of the TM graft was compressed to circumferentially fixate the graft within the holder, which ensured that the printed scaffold infilled with fibrin/collagen matrix, and not the peripheral border region, moved freely during acoustic testing. The $9 \mathrm{~mm}$ aperture of the holder ensures physiologic size measurements of the isolated composite graft. The sound coupler was connected to a sound source (CF1, Tucker-Davis Technologies, Alachua, FL USA) to deliver continuous single tones at selected frequencies and at appropriate stimulus levels to the membrane surface. A calibrated ER-7C probe microphone (Etymotic Research, Elk Grove Village, IL, 
USA) measured sound pressure near the edge of the membrane. DOEH was operated in stroboscopic mode in which nine stroboscopic holograms of the membrane, phase-locked to stimulus phase of $0, \pi / 4, \ldots 2 \pi$, are used to derive the displacement versus time waveform for each point on the membrane surface, and Fourier transformation was used to compute displacement magnitude and phase at each point (Cheng et al., 2010; Del Socorro Hernández-Montes et al., 2009). Measurements were made on six of each PDMS, PLA and PCL printed and in-filled composite grafts, as well as three temporalis fascia specimens using the coupler. The TMs of three fresh human temporal bones with intact middle ear systems were evaluated within its bony annulus.

To increase the amount of reflected light from the TM composite graft, fascia and human TM surface, each was lightly painted with a solution of zinc oxide $(\mathrm{ZnO})$ particles suspended in a sterile saline solution (Acros Organics, Morris Plains, NJ, USA). Application of such paints has been shown to have little effect on the sound-induced displacements of the TM (Cheng et al., 2010; Rosowski et al., 2009).

Mounted TM composite grafts, fascia and the human TM were subjected to continuous tones at four different frequencies across the human range of sound perception: 400, 1000, 3000 and $6000 \mathrm{~Hz}$. Because $3000 \mathrm{~Hz}$ was associated with a narrow-band stimulus artifact affecting holographic measurements of the human $\mathrm{TM}, \mathrm{a}$ $4000 \mathrm{~Hz}$ was used to stimulate the TM. Sound pressure level (SPL) varied from 80-110 $\mathrm{dB}$ at different frequencies to produce DOEH measurable fringes on the membrane surface. The smallest measureable displacement in DOEH is $10-30 \mathrm{~nm}$. The sound levels used in this study are within the linear response range of the human TM and middle ear (Cheng et al., 2010). Displacement of the membrane at each stimulus frequency was 
normalized by the stimulus sound pressure, monitored near the membrane surface. Displacement magnitude and phase were color mapped and compared between TM composite grafts, fascia and the human TM.

\subsection{Laser Doppler Vibrometry}

Laser Doppler vibrometry (LDV) hardware and data acquisition techniques have been described (Aarnisalo et al., 2009; Rosowski et al., 2011). In brief, measurements of the sound-induced velocity of the TM composite grafts, fascia and the human TM at the membrane's center point or umbo are made with an LDV setup. (Polytec OFV 501 fiber interferometer and OFV 2600 vibrometer controller; Polytec Inc., Irvine, CA, USA) The laser was focused on 2-3 $50 \mathrm{~nm}$ diameter polystyrene micro-beads placed at the center of the printed grafts or fascia. The sound stimuli for LDV measurements were broadband chirps containing frequencies of $200 \mathrm{~Hz}$ to $10 \mathrm{kHz}$. The stimuli signals were delivered to the CF1 sound source to produce sound levels between 80 and $120 \mathrm{~dB}$ SPL near the membrane surface. The ER-7C probe microphone was used to monitor SPL near the membrane surface. At each measurement, the LDV and ER-7C microphone response signals to a series of repeated chirps were recorded through an A/D acquisition board running on a PXI system (National Instruments, Austin, TX, USA) and averaged $(n=200)$. The membrane velocity measured by LDV was normalized by the sound pressure monitored by the ER-7C microphone. The sound-induced velocity of the surface of the graft holder or annular bone (in human TM specimens) was used to define a 'noise and artifact' level, which describes the smallest discriminable velocity. Comparison data for human TM umbo velocity in intact temporal bones using the above 
methods was available from our laboratory and has been previously published (Ravicz et al., 2004)

\subsection{Dynamic Mechanical Analysis}

Printed TM composite grafts and fresh human temporalis fascia were subjected to dynamic mechanical analysis (DMA) in tensile mode (Bose $3200, \mathrm{MN}$, USA) to assess stress-relaxation curves. TM composite grafts are mounted using tensile grips grasping the scaffold along radial and circumferential filaments at a set jaw gap of $12 \mathrm{~mm}$. Temporalis fascia measuring $25 \mathrm{~mm} \times 25 \mathrm{~mm}$ is similarly mounted with a jaw gap of $12 \mathrm{~mm}$. Loading force $(\mathrm{N})$ required to cycle the bottom motor through constant sinusoidal displacements of $2 \mathrm{~mm}$ in amplitude at a frequency of $20 \mathrm{~Hz}$ were recorded.

Measurements are conducted over $60 \mathrm{sec}$ to obtain sufficient data to observe any change in mechanical integrity over time. Load was compared between the beginning of the experiment and the end of the experiment to assess stability. TM composite grafts composed of the same scaffold material were compared to identify any mechanical differences in load between the filament arrangements. All studies were carried out at room temperature of $25^{\circ} \mathrm{C}$. In order to replicate conditions in the operating room where fascia is directly harvested and implanted, all materials were tested without preconditioning.

\subsection{Statistical Analysis}

Spearman correlation test was employed to determine the strength of correlation between frequency and graft displacement. Statistical significance was determined by a type I error 
threshold of .05. Statistical analysis was performed on Stata (Statacorp, College Station, TX).

\section{Results}

\subsubsection{D printed tympanic membrane composite grafts}

TM graft scaffolds composed of PDMS, PLA and PCL-based materials were fabricated by DIW in two filamentary configurations: 8 radial $(R)$ and 8 circumferential (C) filaments or 16R and 16C filaments (Fig. 2), each with a total diameter of $25 \mathrm{~mm}$. The filament dimensions are defined by the nozzle diameter, printing speed and applied pressure. Due to modest differences in ink rheology, the printed filament widths for the inner 'mobile' TM region are $209 \pm 9 \mu \mathrm{m}$ for PDMS, $215 \pm 8 \mu \mathrm{m}$ for PLA and $246 \pm 15$ $\mu \mathrm{m}$ for PCL, $(\mathrm{n}=10$ for each print material). TM scaffold thicknesses (orthogonal to the planar ring) prior to infill are $56 \pm 12 \mu \mathrm{m}$ for PDMS, $32 \pm 6 \mu \mathrm{m}$ for PLA and $48 \pm 10 \mu \mathrm{m}$ for PCL. The addition of the infill with collagen / fibrin matrix resulted in a graft thickness of $604 \pm 12 \mu \mathrm{m}$, for all three print materials. For comparison, moist temporalis fascia is approximately $750 \mu \mathrm{m}(\mathrm{n}=3)$ in thickness, and the human TM varies between 50 and $150 \mu \mathrm{m}$ (Kuypers et al., 2006; Van der Jeught et al., 2013).

3.2. Composite grafts demonstrate simple holographic motion at lower frequencies with more complex patterns at frequencies $>1000 \mathrm{~Hz}$

To determine the acoustic properties of specimens, TM composite grafts, fascia and the human TM were evaluated by DOEH to quantify sound induced surface motion patterns (Fig. $3 \& 4$ ). The composite grafts were mounted in the holder (Fig. S1) and controlled sound stimuli used to generate motion. Representative DOEH maps of the 
magnitude of displacement at over 400,000 points on the surface of two PDMS-based composite grafts, temporalis fascia and an intact human TM with middle ear loading are shown in Figure 3. The decibel magnitudes of the displacement normalized by the sound-pressure stimulus ( $\mathrm{dB}$ re $1 \mu \mathrm{m} / \mathrm{Pa}$ ) are coded with progressive colors (color bar to the right of each plot) and standardized within similar range at each frequency (along the column).

In general, DOEH findings illustrate simple modal motion patterns at $400 \mathrm{~Hz}$, with one to three displacement maxima distributed over the entire membrane surface for all three TM composite grafts (Fig. $3 \& 5$ ), the temporalis fascia and the intact TM. The 8C/8R and 16C/16R TM composite grafts result in similar patterns at tested frequencies higher than $1000 \mathrm{~Hz}$, but different patterns at $400 \mathrm{~Hz}$, where the 16C/16R PDMS shows a simpler pattern than the 8C/8R PDMS (Fig. 3) consistent with the dominance of a lower mode of motion (Fletcher, 1992; Tonndorf and Khanna, 1970) in the 16C/16R sample. The patterns in the printed PDMS material show a high degree of radial symmetry especially at the higher frequencies. The temporalis fascia motion patterns are more asymmetric, but generally approximate the TM composite grafts. Human TM motion patterns are highly asymmetric, in part due to the limited membrane displacement at the attachment of the asymmetrically placed manubrium and umbo and the more complicated three-dimensional shape of the human TM (Cheng et al., 2013; Cheng et al., 2010; Khaleghi et al., In press; Khanna and Tonndorf, 1972). The mean magnitude at $400 \mathrm{~Hz}$ of the sound induced motions of three samples of the 4 materials illustrated in Figure 3 varied by a factor of $\sim 5$, with fascia $>$ PDMS $(8 \mathrm{C} / 8 \mathrm{R})>\mathrm{PDMS}$ $(16 \mathrm{C} / 16 \mathrm{R})>$ human TM. 
At higher frequencies $(\geq 1000 \mathrm{~Hz})$ the motions of all the materials compared in Figure 3 become more complex, with multiple areas of maximal displacement separated by regions of reduced displacement. The locations and numbers of the maximal displacement magnitudes vary among the composite grafts, temporalis fascia, and TM, suggesting differences in material properties. At 3000 and $6000 \mathrm{~Hz}$ PDMS grafts and the fascia and TM exhibit 'ordered' motion patterns, with many local displacement maxima and minima arranged alternatively in 'concentric rings' over the membrane surface. In each material, the number of 'rings' increases with increasing frequency. The 16C/16R grafts also showed somewhat smaller and less uniform displacement magnitudes (Fig. 3, Table 1) at the two high frequencies compared to $8 \mathrm{C} / 8 \mathrm{R}$ grafts. Again, the displacement patterns observed in the fascia samples and the human TMs were less symmetric, and particularly noticeable is the reduced displacement over the manubrium in the human TM. The maximum magnitudes of normalized motion in the two PDMS infilled grafts, the fascia and the TM were similar at $3000 \mathrm{~Hz}$, but at $6000 \mathrm{~Hz}$ the maximum motions of the human TMs were on average twice that of fascia and 8 times that of the two PDMS grafts (Table 1).

The phase angle between the displacement and stimulus sound pressure, an indication of the relative timing and directionality of membrane motion, is also measured by DOEH. Figure 4 illustrates phase maps for each of the conditions illustrated in Figure 3. The phase distribution over the membrane surface varies by frequency and graft. At 400 and $1000 \mathrm{~Hz}$ the printed composite grafts exhibit several adjacent areas of opposite phase (light blue with phase $\sim 0$ radians and dark red/dark blue with phases of $+/-\pi$ radians), suggesting these adjacent areas move in the opposite directions. Similar 
patterns are seen on temporalis fascia at 400 and $1000 \mathrm{~Hz}$, while the intact human TM shows smaller variations in phase across its surface, suggesting in-phase motion of much of the TM surface. At higher frequencies, the spatial arrangement of the phase variations matches the complexity of the measured magnitudes (Fig. 3). The fascia shows near half-cycle phase changes between adjacent regions (light blue to dark red/dark blue), which suggests the surface is 'broken up' into regions of opposite motions, though the prominence of the anti-phasic motions decreases as frequency increases. The PDMS composite grafts show regular, but smaller, spatial variations in phase (orange to yellow, or dark red to dark blue, generally $<1$ radian). At $4000 \mathrm{~Hz}$, the intact human TM exhibits mostly in-phase behavior with small regions of anti-phase motion. The regions of anti-phase persist at $6000 \mathrm{~Hz}$, but are of even smaller extent, so that in general sound-induced human TM motion is dominated by regions with small cyclic phase changes that track the changes in magnitude of motion (Fig. 3) (Cheng et al., 2013; Cheng et al., 2010). TM composite grafts fabricated using PLA and PCL have phase angle findings similar to those described for PDMS. (Fig. S2)

In general the patterns of sound-induced motion of TM composite grafts are similar for the three scaffold materials (Fig. 5), though there are clear differences in the number and spacing of local displacement maxima, especially at higher frequencies. We also see variations in the magnitude of the normalized displacements among the three TM composite grafts (Table 1). The mean maximum displacement averaged over three samples is greatest at $400 \mathrm{~Hz}$. PLA demonstrates the greatest displacement magnitude, followed by PCL and then PDMS; this pattern persists with increasing frequency e.g. 1, 3 and $6 \mathrm{kHz}$. PDMS consistently demonstrates a smaller displacement 
when compared to PLA and PCL. There is a statistically significant inverse correlation between frequency and maximum displacement for each of the grafts, fascia and human TM. While maximum displacement differs by TM scaffold print material, the filamentary arrangement, e.g. $8 \mathrm{C} / 8 \mathrm{R}$ versus $16 \mathrm{C} / 16 \mathrm{R}$, has similar averaged maximal graft displacement across tested frequencies (Table 1). While maximal point displacement was similar, evaluation of average surface motion patterns by DOEH (Fig. 3) demonstrates small but noticeable differences in displacement also see in PLA and PCL grafts (data not shown). Temporalis fascia and the intact human TM similarly demonstrated decreased maximum displacement with higher frequency; however, there was high variability (high standard deviation/mean ratio) at each tested frequency for the temporalis fascia group compared to the TM and most composite grafts. The average human TM maximal displacement was small compared to the grafts and fascia at $400 \mathrm{~Hz}$, but was larger than most of the other tested materials at $6000 \mathrm{~Hz}$.

\subsection{TM composite grafts and fascia have similar velocity}

DOEH provides a high spatial resolution image across the entire surface of the membrane, but is limited in its frequency resolution because of the time necessary to measure motion patterns at each frequency. Therefore, only four specific frequencies within the range of human hearing were tested. To determine the acoustic properties of TM composite grafts at a finer frequency resolution, LDV is used to measure sound induced velocity of the center of the membrane across more than 400 frequencies ranging from $0.1 \mathrm{kHz}$ to $10 \mathrm{kHz}$. The mean of velocity measurements on multiple samples of different tested materials, normalized by sound pressure, is displayed in 
Figure 6A. Grafts and fascia are compared to LDV measured average velocity at the umbo of intact human TM specimens from our group's previous work (Ravicz et al., 2004).

The mean of velocity measurements on three different samples of PDMS, PLA, and PCL-based TM composite grafts printed in the $8 \mathrm{C} / 8 \mathrm{R}$ configuration have velocities that are within an order of magnitude of each other with near coincident peaks in the tested frequency range. Velocity is highest at the lowest frequencies tested and appears to peak around $140 \mathrm{~Hz}$ (or lower) at $0.08 \mathrm{~m} / \mathrm{s} / \mathrm{Pa}$. The PDMS composite grafts exhibits the lowest velocities, consistent with the lower displacements observed in this material. Mean velocity of human fascia does not appear similar to 3D printed grafts and is likely a reflection of the wide variability in LDV measured velocity. One of three specimens of human fascia exhibited a similar LDV velocity pattern to averaged composite grafts with similar peaks and a similar shape at high frequency (Fig. 7B). Comparison with intact human TM specimens with an associated middle ear load shows umbo velocity to be much lower than the graft velocities at frequencies less than $1 \mathrm{kHz}$, with a velocity proportional to frequency below $800 \mathrm{~Hz}$ (Ravicz et al., 2004). Mid- and high-frequency umbo velocity is quite similar to the velocity in the center of TM composite grafts and fascia. These data suggests that unloaded grafts have similar acoustic properties to the human TM in mid and high frequencies. When comparing TM composite grafts with $8 \mathrm{C} / 8 \mathrm{R}$ and $16 \mathrm{C} / 16 \mathrm{R}$ designs, (Fig. 6B-D) there is slightly less motion at low frequencies for 16C/16R grafts, although this difference does not appear at mid and high frequencies, and the general similarity suggests the velocity is not significantly affected by the filament count of the graft scaffolds. 
The reproducibility of TM composite graft motion for three different samples of one representative group (PLA 8C/8R) is illustrated in Figure 7A. The patterns and location of local peaks in velocity are consistent across the entire frequency range in the three specimens. This observation is in contrast to the disparate LDV motion of temporalis fascia grafts that were each harvested from three different cadaveric specimens but prepared in an identical fashion. (Fig. 7B)

\subsection{Temporalis fascia has less stability during mechanical testing in contrast to the printed TM composite grafts}

DMA testing at $20 \mathrm{~Hz}$ reveals variable strength and amount of deformation among the printed TM composite grafts and fascia. (Fig.8 A) The choice of printed filament material affects the mechanical properties of the composite grafts. PCL required the highest load, followed by PLA and PDMS. Little change in relaxation is observed in the deformation of the printed grafts during the testing period, with the force required to maintain a constant deformation decreasing by only $10-15 \%$ for each of the three grafts.

(Fig. 8A, B) PLA decreased the least and remained at $90 \%$ of its original value suggesting a greater resistance to stress as compared to the other two materials. By contrast, the force required to maintain a constant deformation of fascia decreases by $70 \%$ over the testing period and is particularly noticeable after several seconds of constant displacement. The 16C/16R designs demonstrate a higher necessary load at the same displacement distance when compared to the $8 \mathrm{C} / 8 \mathrm{R}$ arrangement, suggesting increased filament count increases stiffness of the graft (Fig. 8C). Similar filament count dependent results were also observed in the PCL and PDMS grafts. 


\section{Discussion}

\subsection{Design and Fabrication of the Tympanic Membrane Graft using 3D Printing Techniques}

This study is the first to demonstrate the design, fabrication, and in vitro testing of 3D printed TM grafts. 3D printed TM graft scaffolds can be fabricated on the micron scale with programmable radial and circumferential filament architectures using readily available United States Food and Drug Administration (FDA) approved polymeric materials. The ability to rapidly fabricate and test iterative grafts opens new avenues for novel biocompatible prostheses for TM reconstruction, as well as for the study of specific acoustic and mechanical properties of the human TM.

Given the ultrastructural appearance of the human TM, a biomimetic design for 3D printed TMs was selected based on our current understanding of the modal model for TM motion (Khanna et al., 1972; Rosowski et al., 2011; Tonndorf and Kanna, 1970; Tonndorf and Khanna, 1972). This model suggests that the entire TM surface is equally stimulated by sound pressure, and the motion of the TM surface depends on its shape and mechanical properties, the load of the ossicular chain, and the TM's attachments to the annular ring (Ravicz et al., 2004). Consequently, the global architecture of the TM, including directionality and stiffness of materials, are defining features and should be the basis of any design. Interestingly, recent studies on the mechanics of spider webs with similar radial and circumferential filament orientation indicate that the superior performance of spider silk is due not only to its intrinsic mechanical properties, but also arises from the specific geometrical arrangement of the web (Cranford et al., 2012; Qin 
et al., 2015). These principles may also apply to the fibers of the TM as previous electron microscopy studies have shown the TM's complex radial and circumferential fiber arrangement as fundamental structural features (Knutsson et al., 2009; Lim, 1968; Lim, 1995; Shimada and Lim 1971). Advances in 3D printing techniques now allow production of grafts recapitulating key architectural patterns which can be individually tailored and biomechanically tested.

\subsection{Acoustic and Mechanical Properties of 3D Printed Tympanic Membrane Grafts}

In this study, specific acoustic properties of 3D printed TM grafts, central to the biomechanical function of the TM, were investigated. Spatial displacement patterns and point velocities were evaluated under different fibrous scaffold designs that were manufactured with three different materials. In response to in vitro acoustic testing along the frequency range of human hearing, the magnitude of displacement and point velocity of TM composite grafts are similar in magnitude to fascia velocities, but are also more regular and reproducible than fascia. The PDMS, PCL and PLA-based inks are found to demonstrate consistent, symmetric and regularly varying spatial displacement patterns compared to fascia. The selected print material appears to have a greater influence on spatial displacement patterns than does the tested scaffold arrangements, e.g. $8 \mathrm{C} / 8 \mathrm{R}$ versus $16 \mathrm{C} / 16 \mathrm{R}$.

Mechanical testing of TM grafts revealed desirable properties when compared to human temporalis fascia. The initial load of temporalis fascia is similar to TM composite grafts. Over the course of mechanical testing, however, fascia demonstrates a threefold loss in load (a drop of nearly $70 \%$ ), while TM composite graft load decreases by 
only $10-15 \%$. While instability is a common feature of biologic viscoelastic materials (Fung, 1972; Fung, 1984), these findings are notable as: (1) the significant instability of human temporalis fascia provides one potential explanation for how some surgically placed temporalis fascia thins and retracts over time, and (2) the use of 3D printed TM grafts may offer mechanical advantages over existing graft materials.

Moreover, mechanical testing also demonstrated that grafts with higher filament count resulted in increased load when compared to those with lower filament counts. This may suggest the ability to control graft resistance to load bearing by altering filament count. In this preliminary work, it appears that changes in filament count affect mechanical load, but do not impart significant changes in displacement patterns in response to sound. In the future, TM composite grafts may improve upon the mechanical limitations of existing graft materials without sacrificing acoustic function. Further studies are needed to delineate optimal filament design and orientation to improve sound transmission, while protecting against unwanted graft retraction.

While in vitro testing provides a baseline set of biomechanical data to understand the acoustic properties of fascia and 3D printed grafts, tissue scaffolds may change following in vivo exposure. Prior tissue engineering work has shown that scaffold fiber orientation determines cellular ingrowth patterns, thus final, integrated grafts possess architecture similar to implanted materials (Li et al., 2002) Future studies will need to evaluate differences in acoustic properties before and following in vivo exposure.

\subsection{Comparisons of 3D Printed Tympanic Membrane Grafts to the Native Human} Tympanic Membrane 
Important to the potential clinical translation and future laboratory study, 3D printed grafts also demonstrate qualitative similarities to human TM specimens at high frequencies. Observed high frequency displacement is highly organized with uniform concentric rings for each 3D printed TM graft. These frequency dependent patterns are similarly observed in other human TM studies (Cheng et al., 2013; Cheng et al., 2010). Displacement magnitudes and patters were similar at $3 \mathrm{kHz}$ and $6 \mathrm{kHz}$, indicating grafts have basic acoustic properties, in the high frequencies, that replicate human TM motion. The angle of phase variations of 3D printed TMs are also similar to that observed at mid to high frequencies in human and animal TMs (Cheng et al., 2013; Cheng et al., 2010; Rosowski et al., 2011; Rosowski et al., 2009).

A significant consideration in these comparisons is that 3D printed TM grafts are measured in an isolated state, while the measurements in the intact TM / middle ear involves the coupling of the ossicular chain and the fluid filled cochlea. As seen in the results of our study, the ossicular chain and cochlear load to the TM is known to reduce TM motion at frequencies below $1-2 \mathrm{kHz}$ but have little effect at higher frequencies (Ulku et al., 2014). The addition of this load to the intact TM, may explain the differences observed in the surface motion and TM velocity at frequencies below $2 \mathrm{kHz}$. In addition, the conical shape of the TM may also account for differences in holographic results. Future research will incorporate coupling of conical 3D printed grafts to a representative middle ear load.

Human TM point velocity in response to sound has also previously been studied using LDV. Point velocity at the center of TM composite grafts exhibit different pattern peaks when compared to human TM umbo velocity (Cheng et al., 2013; Cheng et al., 
2010; Rosowski et al., 2011; Rosowski et al., 2009). This difference is expected given the middle ear load associated with an intact ossicular chain. Importantly, the variability in point velocity between identically manufactured 3D composite grafts is small, indicating consistent acoustic properties. This stands in contrast to the wide observed variability in LDV measured TM velocity of anatomically matched ears following cartilage tympanoplasty (Aarnisalo et al., 2009; Cheng et al., 2010). Taken together, DOEH and LDV measurements of TM composite grafts provide consistent, expected results when compared to prior findings of the human TM.

\subsection{Limitations of 3D Printed Grafts}

There are several limitations to our study. The design and fabrication of 3D printed TM composite grafts is in the early phases of development. We selected three experimental polymers (PDMS, PCL, and PLA) and two relatively simple radial and circumferential filamentary scaffold designs. We recognize that the human TM possesses a significantly more complex ultrastructure. For example, the attachment of the TM to the malleus is purposefully not included in designs as the utility of such a structure in a biomimetic graft is as yet unclear. Surgical placement of 3D printed TM grafts will require consideration of graft orientation to allow for a reliable connection to the ossicular chain. Finally, the testing performed during this investigation was done in vitro. It is difficult to predict whether these results would be equivalent if the grafts were incorporated into living tissue. As previously described, fascia is remodeled following implantation (Szabo, 2006), and similar remodeling may occur with 3D printed grafts. 
Nevertheless, these initial in vitro studies establish a baseline for future in vivo comparison.

We urge caution on calling a construct a "biomimetic" TM without demonstration of functional data, such as from acoustic or mechanical tests. As an analogy, the creation of a biomimetic lens for vision would be incomplete without an understanding of its basic optical properties. Viability of a biomimetic TM graft necessitates at a minimum acoustic data as the graft may have limited movement in response to sound frequencies and amplitudes within range of human hearing. One recent study reported on 3D printed scaffolds with design features similar to the human TM (Mota et al., 2015). Employing poly (lactic-co-glycolic acid), poly (ethylene oxide terephthalate)/poly (butylene terephthalate) block copolymer, and an electrospinning technique, Mota et al. created a scaffold and pore arrangement trilayer membrane. This work reflects a significant step towards demonstrating the fabrication of a TM-like scaffold. There is no description, however, of functional data beyond cell studies. Without understanding the graft's acoustic or mechanical properties, it is difficult to interpret the physiologic relevance of the results. Nevertheless, Mota et al. demonstrate a compelling method to create a TMlike structure using manufacturing techniques that may be readily coupled to functional assays.

\subsection{Potential Application of 3D Printed Grafts to Isolate Structural Features of Human}

\section{Tympanic Membrane}

Finally, beyond the potential for use as graft material in human tympanoplasty procedures, the ability to control the design of a TM-like structure has implications for 
the study of middle ear mechanics. Many unanswered questions remain regarding the function of the TM and its role in the regulation of sound transmission to the middle ear. In particular, there are a host of biophysical models, such as modal and traveling wave models, that attempt to explain the motion of the TM at low and high frequencies (Aarnisalo et al., 2010; Cheng et al., 2010; Dobrev et al., 2015; Khaleghi et al., 2015;

Khaleghi et al., 2013; Rosowski et al., 2011). The ability to fine-tune the ultrastructure to control for specific properties of the TM may enable further understanding of the nuances of TM motion and provide experimental designs to test finite element models (Gan and Wang, 2007; Gan et al., 2004).

\section{Conclusion}

This study demonstrates the design, fabrication, and preliminary in-vitro evaluation of 3D printed TM grafts. Methodology in the manufacture and testing of grafts may provide greater understanding of the biomechanical properties of the human TM, as well as future development of a biomimetic TM graft. 


\section{Acknowledgments}

We would like to thank Kimberly Homan, Ryan Truby, David Kolesky, Mark Scott, Alex Valentine, and Michelle Walsh of the Wyss Institute for Biologically Inspired Engineering for their support of this project. We would like to thank Rosh K. Sethi, MD, MPH for expert statistical analysis support. We would like to acknowledge Louise Collins of the Leroy A Schall Library of Otolaryngology at Massachusetts Eye and Ear Infirmary. N. Black and J. Lewis would like to acknowledge support from the National Science Foundation Graduate Research Fellowship, the Wyss Institute, and the Army Research Office. 


\section{References}

Aarnisalo, A., Cheng, J., Ravicz, M., Hulli, N., Harrington, E., Hernandez-Montes, M., Furlong, C., Merchant, S., Rosowski, J. 2009. Middle ear mechanics of cartilage tympanoplasty evaluated by laser holography and vibrometry. Otol Neurotol. 30, 1209-14.

Aarnisalo, A.A., Cheng, J.T., Ravicz, M.E., Furlong, C., Merchant, S.N., Rosowski, J.J. 2010. Motion of the tympanic membrane after cartilage tympanoplasty determined by stroboscopic holography. Hear Res 263, 78-84.

Barry, R., Shepherd, R., Hanson, J., Nuzzo, R., Wiltzius, P., Lewis, J. 2009. Direct-Write Assembly of 3D Hydrogel Scaffolds for Guided Cell Growth. Advanced Materials 21, 2407-2410.

Boedts, D., De Cock, M., Andries, L., Marquet, J. 1990. A scanning electronmicroscopic study of different tympanic grafts. Am J Otol 11, 274-7.

Cabra, J., Monux, A. 2010. Efficacy of cartilage palisade tympanoplasty: randomized controlled trial. Otology \& neurotology : official publication of the American Otological Society, American Neurotology Society [and] European Academy of Otology and Neurotology 31, 589-95.

Calobrace, M. 2014. The design and engineering of the MemoryShape breast implant. Plast Reconstr Surg. 134, 10S-5S.

Cheng, J., Hamade, M., Merchant, S., Rosowski, J., Harrington, E., Furlong, C. 2013. Wave motion on the surface of the human tympanic membrane: holographic measurement and modeling analysis. J Acoust Soc Am 133, 918-37.

Cheng, J., Aarnisalo, A., Harrington, E., Hernandez-Montes Mdel, S., Furlong, C., Merchant, S., Rosowski, J. 2010. Motion of the surface of the human tympanic membrane measured with stroboscopic holography. Hear Res. 263, 66-77.

Chu, C., Coutts, R., Yoshioka, M., Harwood, F., Monosov, A., Amiel, D. 1995. Articular cartilage repair using allogeneic perichondrocyte-seeded biodegradable porous polylactic acid (PLA): a tissue-engineering study. J Biomed Mater Res 29, 114754.

Cranford, S., Tarakanova, A., Pugno, N., Buehler, M. 2012. Nonlinear material behaviour of spider silk yields robust webs. Nature 482, 72-6.

Decraemer, W., Dirckx, J., Funnell, W. 1991. Shape and derived geometrical parameters of the adult, human tympanic membrane measured with a phaseshift moire interferometer. Hear Res. 51, 107-21.

Del Socorro Hernández-Montes, M., Furlong, C., Rosowski, J., Hulli, N., Harrington, E., Cheng, J., Ravicz, M., Santoyo, F. 2009. Optoelectronic holographic otoscope for 
measurement of nano-displacements in tympanic membranes. J Biomed Opt 14, 034023.

Dhatta, R. 1995. Technological and economic potential of poly(lactic acid) and lactic acid derivatives. . FEMS Microbiology Reviews 16, 221-231.

Dobrev, I., Furlong, C., Cheng, J.T., Rosowski, J.J. 2015. Optimization of a lensless digital holographic otoscope system for transient measurements of the human tympanic membrane. Exp Mech 55, 459-470.

Eviatar, A. 1978. Tragal perichondrium and cartilage in reconstructive ear surgery. Laryngoscope 88, Suppl 11: 1-23.

Fletcher, N. 1992. Acoustic Systems in Biology Oxford University Press, New York.

Fung, Y. 1972. Stress-strain history relations of soft tissues in simple elongation. Prentice-Hall, Englewood Cliffs.

Fung, Y. 1984. Structure and Stress-Strain Relationship of Soft Tissues. Am Zool 24, 13-22.

Galow, T., Drechsler, U., Hanson, J., Rotello, V. 2002. Highly reactive heterogeneous Heck and hydrogenation catalysts constructed through 'bottom-up' nanoparticle self-assembly. Chem Commun 21, 1076-7.

Gan, R., Wang, X. 2007. Multifield coupled finite element analysis for sound transmission in otitis media with effusion. J Acoust Soc Am 122, 3527-38.

Gan, R., Feng, B., Sun, Q. 2004. Three-dimensional finite element modeling of human ear for sound transmission. Ann Biomed Eng 32, 847-59.

Geckil, H., Xu, F., Zhang, X., Moon, S., Demirci, U. 2010. Engineering hydrogels as extracellular matrix mimics. Nanomedicine (Lond) 5, 469-84.

Gratson, G., Xu, M., Lewis, J. 2004. Microperiodic structures: direct writing of threedimensional webs. Nature 428, 386.

Hanson Shepherd, J., Parker, S., Shepherd, R., Gillette, M., Lewis, J., Nuzzo, R. 2011. 3D Microperiodic Hydrogel Scaffolds for Robust Neuronal Cultures. Adv Funct Mater 21, 47-54.

Hardman, J., Muzaffar, J., Nankivell, P., Coulson, C. 2015. Tympanoplasty for Chronic Tympanic Membrane Perforation in Children: Systematic Review and Metaanalysis. Otol Neurotol. 36, 796-804.

Hardy, J., Amend, M., Geissler, S., Lynch, V., Schmidt, C. 2015. Peptide-directed assembly of functional supramolecular polymers for biomedical applications: 
electroactive molecular tonguetwisters (oligoalanine-oligoaniline-oligoalanine) for electrochemically enhanced drug delivery. J Mater Chem 3, 5005-9.

Hiraide, F., Sawada, M., Inouye, T., Miyakogawa, N., Tsubaki, Y. 1980. The fiber arrangement of the pathological human tympanic membrane. Arch Otorhinolaryngol 226, 93-9.

House, W., Patterson, M., Linthicum, F.J. 1966. Incus homografts in chronic ear surgery. Arch Otolaryngol 84, 148-53.

Kaylie, D., Gardner, E., Jackson, C. 2006. Revision chronic ear surgery. Otolaryngol Head Neck Surg 134, 443-50.

Khaleghi, M., Cheng, J., Furlong, C., Rosowski, J. In press. Four-dimensional measurements of the in-plane and out-of-plane motions of the human tympanic membrane. . J. Acoust. Soc . Am.

Khaleghi, M., Furlong, C., Ravicz, M., Cheng, J.T., Rosowski, J.J. 2015. Threedimensional vibrometry of the human eardrum with stroboscopic lensless digital holography. J Biomed Opt 20, 051028.

Khaleghi, M., Lu, W., Dobrev, I., Cheng, J.T., Furlong, C., Rosowski, J.J. 2013. Digital holographic measurements of shape and 3D sound-induced displacements of Tympanic Membrane. Opt Eng 52, 101916.

Khanna, S., Tonndorf, J. 1972. Tympanic membrane vibrations in cats studied by timeaveraged holography. J Acoust Soc Am 51, 1904-1920.

Knutsson, J., Bagger-Sjoback, D., von Unge, M. 2009. Collagen type distribution in the healthy human tympanic membrane. Otol Neurotol. 30, 1225-9.

Kohn, F., Feijen, J., Feenstra, L. 1984. New perspectives in myringoplasty. . Int J Artif Organs. 7, 151-62.

Kulkarni, R., Moore, E., Hegyeli, A., Leonard, F. 1971. Biodegradable poly(lactic acid) polymers. J Biomed Mater Res 5, 169-81.

Kuypers, L., Decraemer, W., Dirckx, J. 2006. Thickness distribution of fresh and preserved human eardrums measured with confocal microscopy. Otol Neurotol. 27, 256-64.

Levin, B., Rajkhowa, R., Redmond, S., Atlas, M. 2009. Grafts in myringoplasty: utilizing a silk fibroin scaffold as a novel device. Expert Rev Med Devices. 2009 6, 65364.

Lewis, J. 2006. Direct Ink Writing of 3D Functional Materials. Advanced Functional Materials 16, 2193-2204. 
Li, W.J., Laurencin, C.T., Caterson, E.J., Tuan, R.S., Ko, F.K. 2002. Electrospun nanofibrous structure: a novel scaffold for tissue engineering. J Biomed Mater Res 60, 613-21.

Lim, D. 1968. Tympanic membrane. Electron microscopic observation. I: pars tensa. Acta oto-laryngologica 66, 181-98.

Lim, D. 1995. Structure and function of the tympanic membrane: a review. Acta otorhino-laryngologica Belgica 49, 101-15.

Lin, C., Yeh, Y., Lin, W., Yang, M. 2014. Novel silicone hydrogel based on PDMS and PEGMA for contact lens application. Colloids Surf B Biointerfaces. 123, 986-94.

Lukasiak, H., Dorosz, A., Prokopowicz, M., Rosciszewski, P., Falkiewicz, B. 2005. Biodegradation of Silicones (Organosiloxanes).

Lyons, S.A., Su, T., Vissers, L.E., Peters, J.P., Smit, A.L., Grolman, W. 2015. Fascia compared to one-piece composite cartilage-perichondrium grafting for tympanoplasty. Laryngoscope.

Marquet, J. 1971. Human middle ear transplants. J Laryngol Otol 85, 523-539.

Mironov, V., Boland, T., Trusk, T., Forgacs, G., Markwald, R.R. 2003. Organ printing: computer-aided jet-based 3D tissue engineering. Trends in Biotechnology 21, 157-161.

Monasta, L., Ronfani, L., Marchetti, F., Montico, M., Vecchi Brumatti, L., Bavcar, A., Grasso, D., Barbiero, C., Tamburlini, G. 2012. Burden of disease caused by otitis media: systematic review and global estimates. PLoS One 7, e36226.

Mota, C., Danti, S., D'Alessandro, D., Trombi, L., Ricci, C., Puppi, D., Dinucci, D., Milazzo, M., Stefanini, C., Chiellini, F., Moroni, L., Berrettini, S. 2015. Multiscale fabrication of biomimetic scaffolds for tympanic membrane tissue engineering. Biofabrication 7, 025005.

Murphy, S.V., Atala, A. 2014. 3D bioprinting of tissues and organs. Nat Biotechnol 32, 773-85.

O'Connor, K.N., Tam, M., Blevins, N.H., Puria, S. 2008. Tympanic membrane collagen fibers: a key to high-frequency sound conduction. Laryngoscope 118, 483-90.

Pfaltz, C., Griesemer, C. 1985. Pericard: a new biometerial for tympanoplasty. Preliminary report. Am J Otolaryngol 6, 266-268.

Qin, Z., Compton, B., Lewis, J., Buehler, M. 2015. Structural optimization of 3D-printed synthetic spider webs for high strength. Nature communications 6, 7038. 
Ravicz, M.E., Rosowski, J.J., Merchant, S.N. 2004. Mechanisms of hearing loss resulting from middle-ear fluid. Hearing research 195, 103-30.

Rosowski, J., Cheng, J., Merchant, S., Harrington, E., Furlong, C. 2011. New data on the motion of the normal and reconstructed tympanic membrane. Otol Neurotol. 32, 1559-67.

Rosowski, J., Cheng, J., Ravicz, M., Hulli, N., Hernandez-Montes, M., Harrington, E., Furlong, C. 2009. Computer-assisted time-averaged holograms of the motion of the surface of the mammalian tympanic membrane with sound stimuli of 0.4-25 kHz. Hear Res. 253, 83-96.

Rubin, R. 1982. The disease in society-evaluation of chronic otitis media in general and cholesteatoma in particular., Amsterdam.

Seliktar, D. 2012. Designing cell-compatible hydrogels for biomedical applications. Science 336, 1124-8.

Sheehy, J.L., Anderson, R.G. 1980. Myringoplasty. A review of 472 cases. The Annals of otology, rhinology, and laryngology 89, 331-4.

Shimada, T., Lim, D. 1971. The fiber arrangement of the human tympanic membrane. A scanning electron microscopic observation. Ann Otol Rhinol Laryngol. 80, 210-7.

Smith, A. 1986. Evaluation of poly (lactic acid) as a biodegradable drug delivery system for parenteral administration. Int J Pharm 30, 215-220.

Strens, D., Knerer, G., Van Vlaenderen, I., Dhooge, I. 2012. A pilot cost-of-illness study on long-term complications/sequelae of AOM. B-ENT 8, 153-65.

Sun, L., Parker, S., Syoji, D., Wang, X., Lewis, J., Kaplan, D. 2012. Direct-Write Assembly of 3D Silk/hydroxyapatite Scaffolds for Bone Co-Cultures. Advanced Healthcare Materials 1, 729-735.

Szabo, L.Z. 2006. How can an underlaid fascia graft form the middle layer of a reconstructed tympanic membrane? Laryngoscope 116, 1674-7.

Tonndorf, J., Khanna, S. 1970. The role of the tympanic membrane in middle ear transmission. Ann Otol 79, 743-753.

Tonndorf, J., Khanna, S. 1972. Tympanic-membrane vibrations in human cadaver ears studied by time- averaged holography. J Acoust Soc Am 52, 1221-1233.

Uebersax, L., Apfel, T., Nuss, K., Vogt, R., Kim, H., Meinel, L., Kaplan, D., Auer, J., Merkle, H., von Rechenberg, B. 2013. Biocompatibility and osteoconduction of macroporous silk fibroin implants in cortical defects in sheep. Eur J Pharm Biopharm 85, 107-18. 
Ulku, C., Cheng, J., Guignard, J., Rosowski, J. 2014. Comparisons of the mechanics of partial and total ossicular replacement prostheses with cartilage in a cadaveric temporal bone preparation. Acta Otolaryngol. 134, 776-84.

Van der Jeught, S., Dirckx, J.J., Aerts, J.R., Bradu, A., Podoleanu, A.G., Buytaert, J.A. 2013. Full-field thickness distribution of human tympanic membrane obtained with optical coherence tomography. Journal of the Association for Research in Otolaryngology : JARO 14, 483-94. 
Table 1: Mean maximum normalized displacement measured by Digital Opto-Electronic Holography (Standard Deviation).

\begin{tabular}{|c|c|c|c|c|c|c|c|c|}
\hline & & \multicolumn{7}{|c|}{ Frequency tested } \\
\hline & \multicolumn{2}{|c|}{$400 \mathrm{~Hz}$} & \multicolumn{2}{|c|}{$1000 \mathrm{~Hz}$} & \multicolumn{2}{|c|}{$3000 \mathrm{~Hz}$} & \multicolumn{2}{|c|}{$6000 \mathrm{~Hz}$} \\
\hline $\begin{array}{c}\text { Scaffold (circumferential } x \\
\text { radial) }\end{array}$ & $8 \times 8$ & $16 \times 16$ & $8 \times 8$ & $16 \times 16$ & $8 \times 8$ & $16 \times 16$ & $8 \times 8$ & $16 \times 16$ \\
\hline PDMS (n=3) & $\begin{array}{c}1.88 \\
(0.41)\end{array}$ & $\begin{array}{c}1.30 \\
(0.09)\end{array}$ & $\begin{array}{c}0.44 \\
(0.03)\end{array}$ & $\begin{array}{c}0.37 \\
(0.13)\end{array}$ & $\begin{array}{c}0.14 \\
(0.06)\end{array}$ & $\begin{array}{c}0.11 \\
(0.01)\end{array}$ & $\begin{array}{c}0.010 \\
(0.005)\end{array}$ & $\begin{array}{c}0.009 \\
(0.002)\end{array}$ \\
\hline $\operatorname{PLA}(n=3)$ & $\begin{array}{c}5.66 \\
(1.83)\end{array}$ & $\begin{array}{l}6.40 \\
(1.7)\end{array}$ & $\begin{array}{c}1.25 \\
(0.41)\end{array}$ & $\begin{array}{c}1.64 \\
(0.78)\end{array}$ & $\begin{array}{c}0.27 \\
(0.05)\end{array}$ & $\begin{array}{c}0.40 \\
(0.05)\end{array}$ & $\begin{array}{c}0.033 \\
(0.011)\end{array}$ & $\begin{array}{c}0.060 \\
(0.002)\end{array}$ \\
\hline $\mathrm{PCL}(\mathrm{n}=3)$ & $\begin{array}{c}4.05 \\
(1.47) \\
\end{array}$ & $\begin{array}{c}4.08 \\
(1.78)\end{array}$ & $\begin{array}{c}1.41 \\
(0.53)\end{array}$ & $\begin{array}{c}3.11 \\
(3.13) \\
\end{array}$ & $\begin{array}{c}0.41 \\
(0.07) \\
\end{array}$ & $\begin{array}{c}0.38 \\
(0.04) \\
\end{array}$ & $\begin{array}{c}0.03 \\
(0.007) \\
\end{array}$ & $\begin{array}{c}0.130 \\
(0.110)\end{array}$ \\
\hline Temporalis Fascia $(n=3)$ & \multicolumn{2}{|c|}{$\begin{array}{c}4.63 \\
(3.37)\end{array}$} & \multicolumn{2}{|c|}{$\begin{array}{c}0.78 \\
(0.49)\end{array}$} & \multicolumn{2}{|c|}{$\begin{array}{c}0.15 \\
(0.16)\end{array}$} & \multicolumn{2}{|c|}{$\begin{array}{c}0.037 \\
(0.039)\end{array}$} \\
\hline Human TM $(n=3)$ & \multicolumn{2}{|c|}{$\begin{array}{c}0.94 \\
(0.60)\end{array}$} & \multicolumn{2}{|c|}{$\begin{array}{c}1.12 \\
(0.91)\end{array}$} & \multicolumn{2}{|c|}{$\begin{array}{l}{ }^{* *} 0.09 \\
(0.09)\end{array}$} & \multicolumn{2}{|c|}{$\begin{array}{c}0.082 \\
(0.032)\end{array}$} \\
\hline
\end{tabular}

${ }^{*}$ The units of normalized displacement are $\mu \mathrm{m} / \mathrm{Pa}$.

** $4000 \mathrm{~Hz}$ was used for human TM because $3000 \mathrm{~Hz}$ is associated with a narrow-band stimulus artifact Note: Statistically significant inverse correlation between frequency and maximum displacement: $8 \times 8$ PDMS ( $p<0.0001), 8 \times 8$ PLA ( $p<0.0001), 8 \times 8$ PCL ( $<<0.0001) ; 16 \times 16$ PDMS $(p<0.0001), 16 \times 16$ PLA $(p<0.0001), 16 \times 16 \mathrm{CL}(p<0.0003)$; fascia $(p<0.0001)$ and human TM $(p<0.0045)$. 


\section{Figure Legends}

Fig. 1. 3D printing of tympanic membrane composite grafts.

(A) Multi-material 3D printing apparatus. (B) Layered circumferential and radial filaments are printed first using a $100 \mu \mathrm{m}$ nozzle. (C) The same material (with blue colorant) is then printed to create the outer border region. (D) The printed and cured scaffold is then infilled with $100 \mu \mathrm{L}$ of fibrin/collagen hydrogel matrix. (E) Final composite graft with fibrin/collagen matrix.

Fig. 2. Printed tympanic membrane scaffolds with different materials and designs. (A-C) Images of TM scaffolds composed of PDMS, PLA, and PCL filaments, respectively, with $8 \mathrm{C} / 8 \mathrm{R}$ and $16 \mathrm{C} / 16 \mathrm{R}$ filamentary architectures. The TMs in the first column of each box have a total diameter of $25 \mathrm{~mm}$. The next two columns show higher magnification images, 50x with a scale bar of $1 \mathrm{~mm}$ and $100 x$ with a scale bar of 500 $\mu \mathrm{m}$, respectively. (D) Image of a representative printed scaffold highlighting design features.

Fig. 3. Digital opto-electronic holography (DOEH) fringe patterns of PDMS-based tympanic membrane composite grafts, human fascia and tympanic membrane controls.

Top row shows the displacement patterns of the visible $9 \mathrm{~mm}$ diameter section of an 8C/8R PDMS graft, the second row a 16C/16R PDMS graft, the third row a sheet of fresh human temporalis fascia, the fourth row a human TM with intact middle ear. The four columns show measurements at different frequencies. Because $3000 \mathrm{~Hz}$ was associated with a narrow-band stimulus artifact affecting holographic measurements of the human TM, $4000 \mathrm{~Hz}$ was used with the TM sample. Color bars are standardized at each frequency. Displacement is normalized by sound pressure, and units are $\mathrm{dB}$ re 1 $\mu \mathrm{m} / \mathrm{Pa}$.

Fig. 4. Digital Opto-electronic holography (DOEH) measured phase angle of tympanic membrane composite grafts compared to control samples Representative DOEH images comparing the phase angle of the displacement relative to sound pressure in radians for PDMS-based TM composite grafts (top two rows), the third row a sheet of fresh human temporalis fascia, the fourth row a human TM with intact middle ear. The color bar codes the phase in radians from $+\pi$ (dark red) to $-\pi$ (dark blue). Because of the circularity of phase $+\pi$ is equivalent to $-\pi$, and regions coded in dark red and dark blue are nearly identical in phase.

Fig. 5. Digital opto-electronic holography (DOEH) fringe patterns of tympanic membrane grafts fabricated with different materials.

DOEH results from TM composite grafts consisting of PDMS (top row), PLA (middle row) and PCL (bottom row) all with $8 \mathrm{C} / 8 \mathrm{R}$ architecture are shown. Color bars are standardized at each frequency, displacement is normalized by sound pressure and units are $\mathrm{dB}$ re $1 \mu \mathrm{m} / \mathrm{Pa}$. 
Fig. 6. Velocity normalized by stimulus sound pressure of tympanic membrane composite grafts, fascia and the human TM across the human frequency range (A) Laser Doppler vibrometry measured mean velocity for three specimens of $8 \mathrm{C} / 8 \mathrm{R}$ TM composite grafts of varying composition (PDMS, PLA, and PCL), fascia and human TMs with intact middle ears. (B-D) Comparisons of PDMS, PLA and PCL-based TM composite grafts with different designs (8C/8R and 16C/16R). Human TM measurements were performed in our laboratory under identical testing conditions, the results of which have been previously published.(Ravicz et al., 2004).

Fig. 7. Differences in normalized surface velocity among PLA-based tympanic membrane composite grafts and fascia. (A) Three separate fabricated 8C/8R PLA TM composite grafts have uniform surface velocity as measured by laser Doppler vibrometry. Velocity peaks are highly consistent. PDMS and PCL also have consistent velocities among graft samples. (Data not shown.) (B) Three separate human temporalis fascia samples harvested and prepared identically yield distinctly different velocity patterns.

Fig. 8. Dynamic mechanical analysis (DMA) for tympanic membrane composite grafts and fascia (A) Representative first and last of 60 DMA cycles comparing different graft materials and fascia. All TM composite materials demonstrate resiliency over time, with the force required for a $2 \mathrm{~mm}$ displacement falling by $10-15 \%$ between the first and $60^{\text {th }}$ measurement cycle, respectively. The fascia, on the other hand, is more unstable, with the force needed to displace it falling by $70 \%$. (B) Stress-relaxation curves showing force required for a $2 \mathrm{~mm}$ displacement over the entire testing cycle demonstrates the resiliency of TM composite materials (PCL) versus the instability of fascia. (C) Representative difference in mechanical rigidity for $8 \mathrm{C} / 8 \mathrm{R}$ grafts when compared to $16 \mathrm{C} / 16 \mathrm{R}$ grafts. Note that a larger force is required to displace the graft a fixed distance in higher filament count grafts. This suggests high filament counts are associated with a more mechanically rigid graft. 


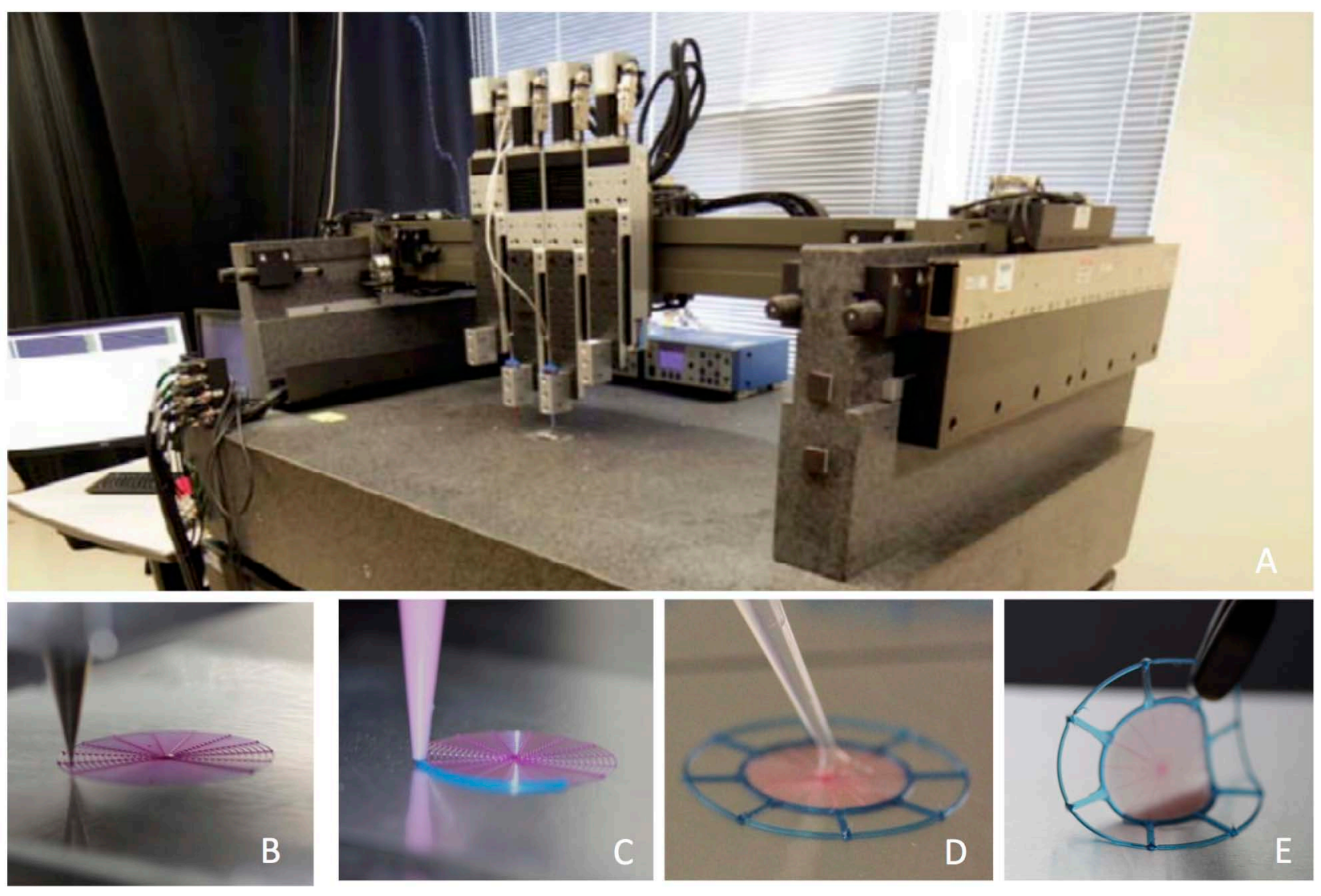


Frequency

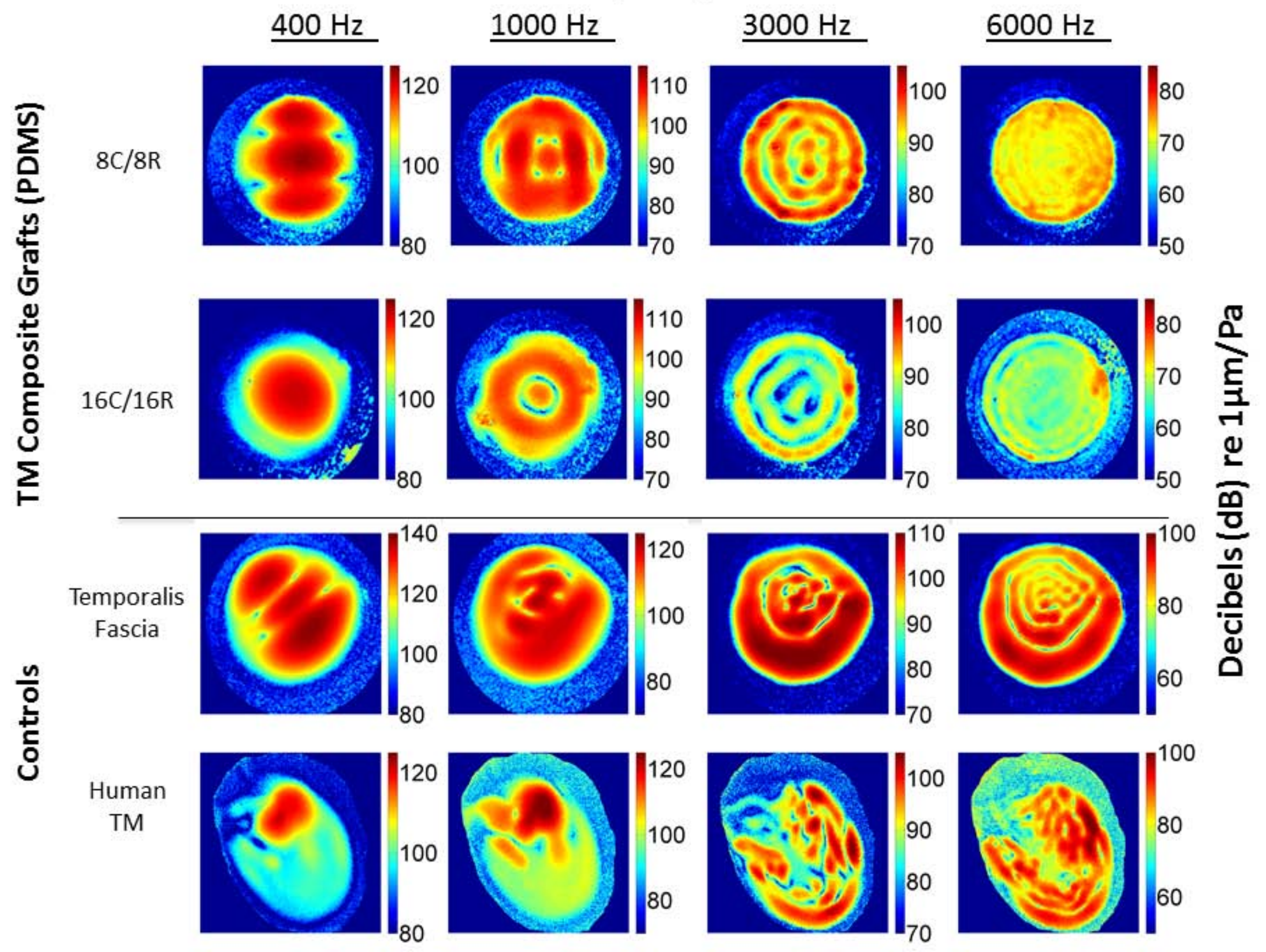




\section{Frequency}

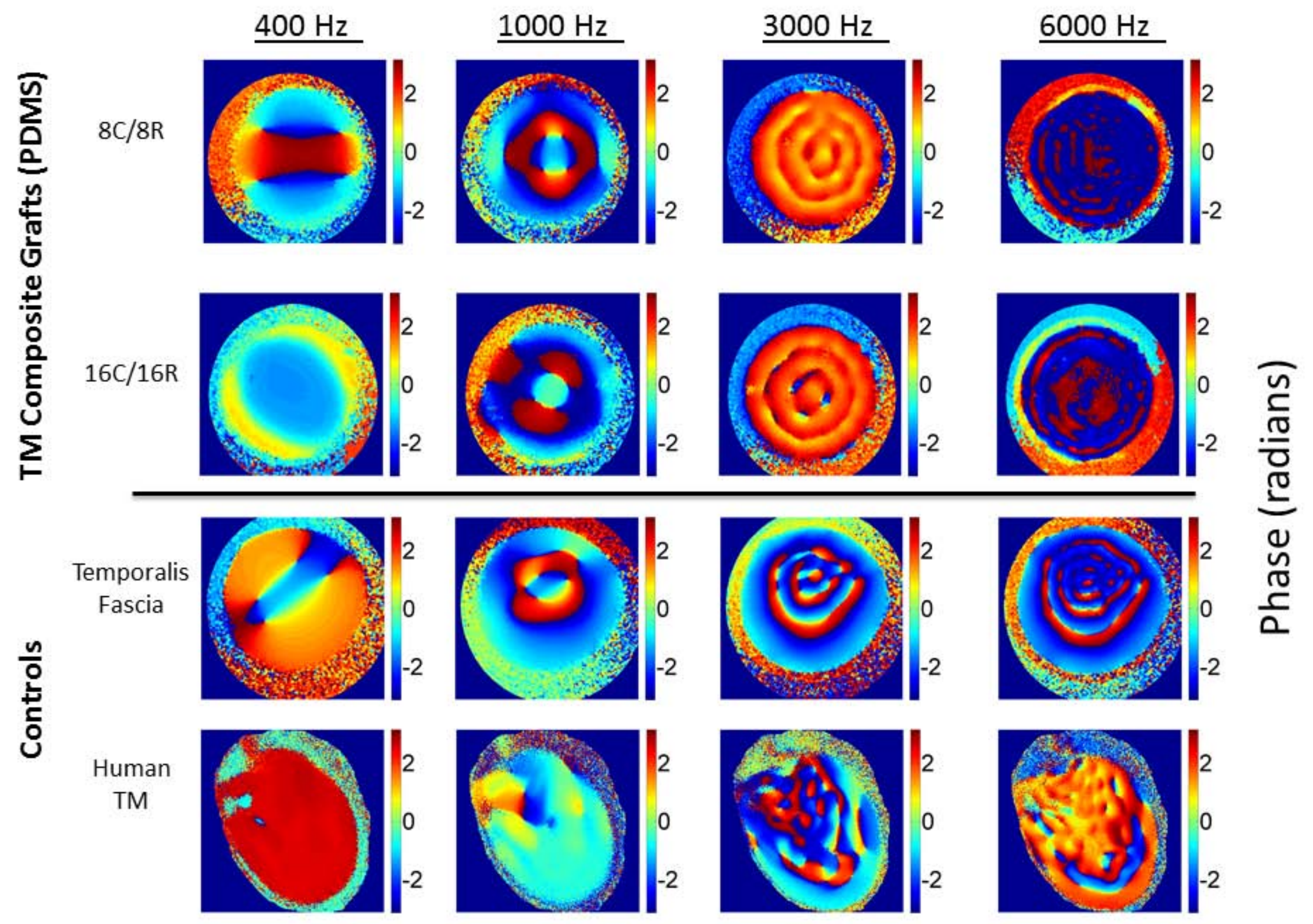




\section{Frequency}

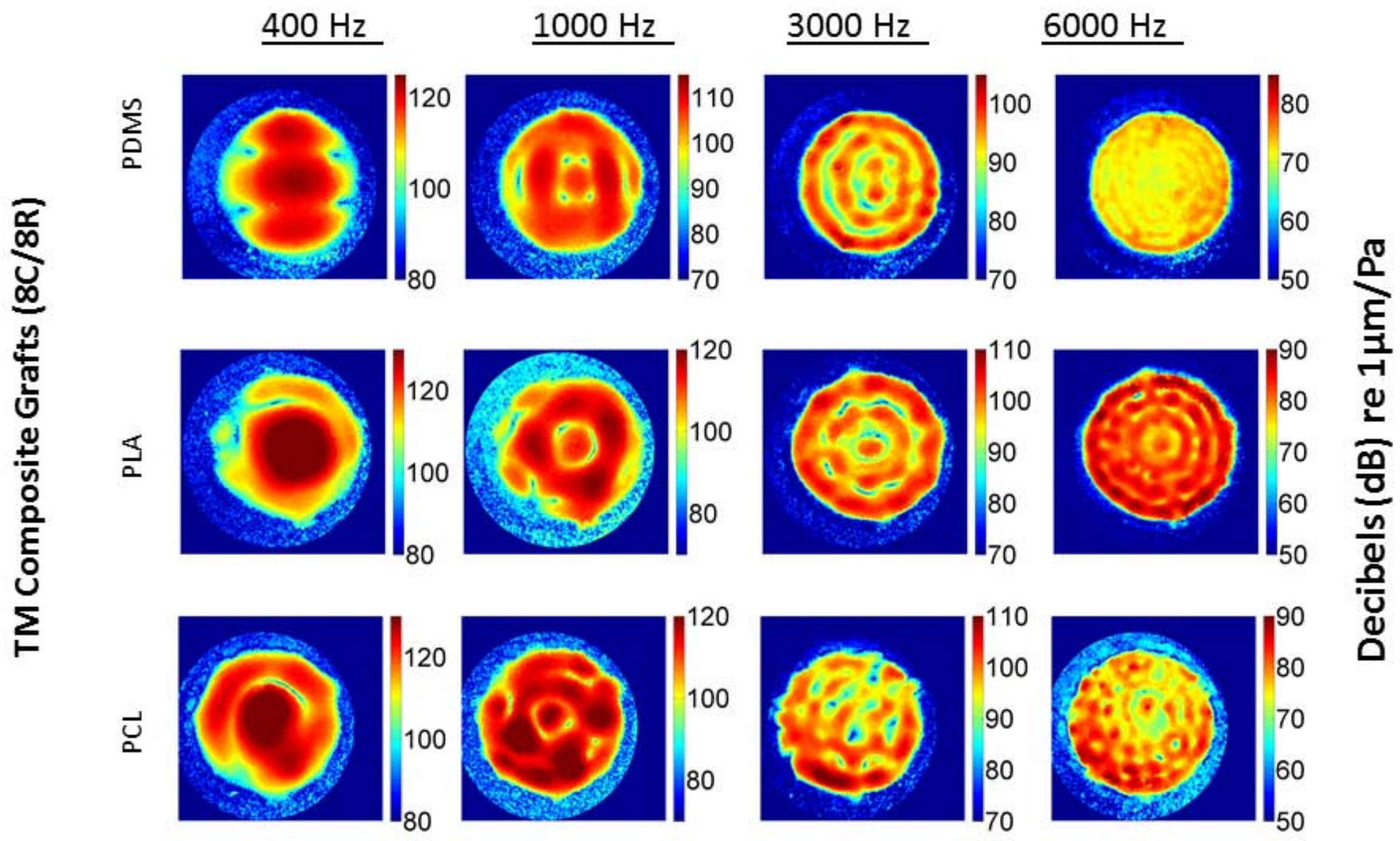


All Materials

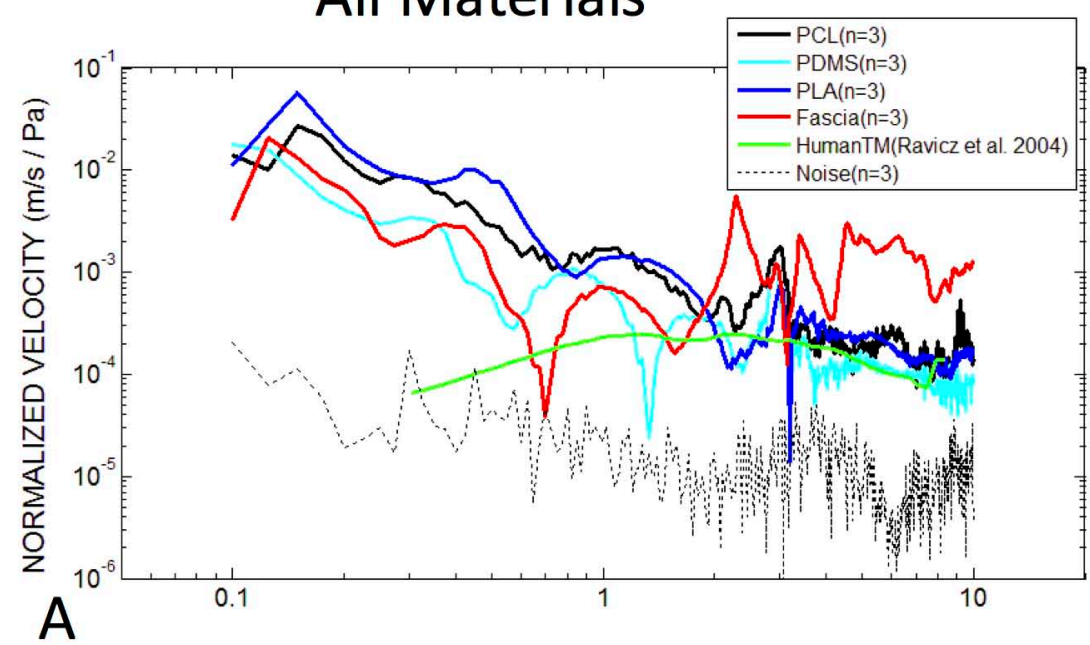

PLA

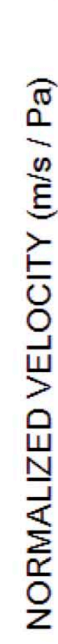
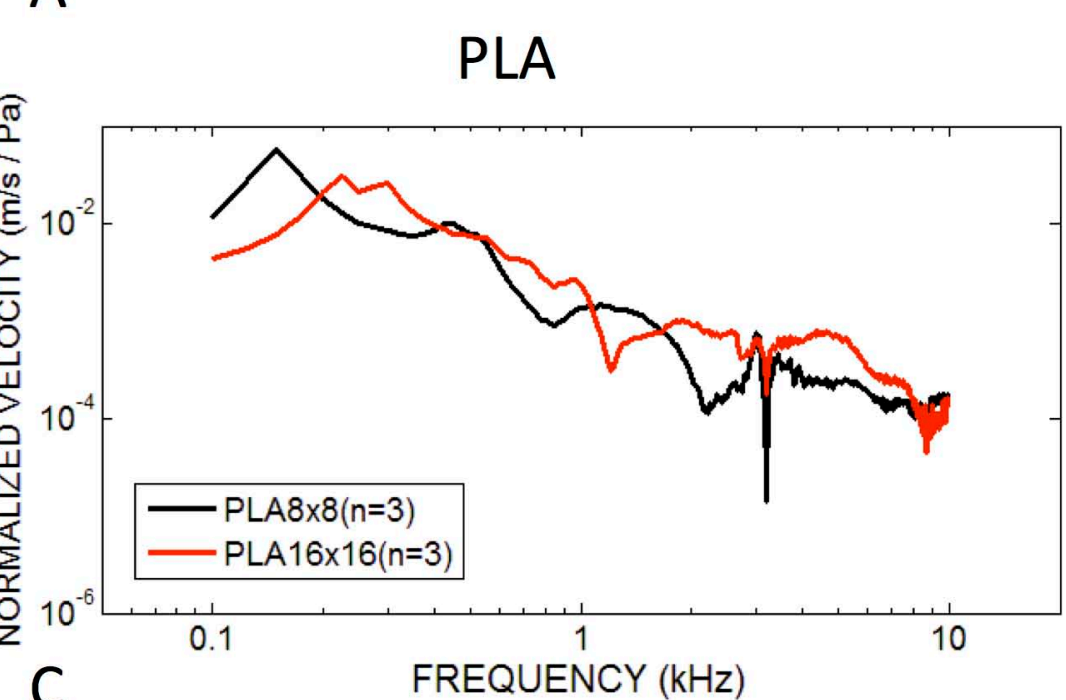

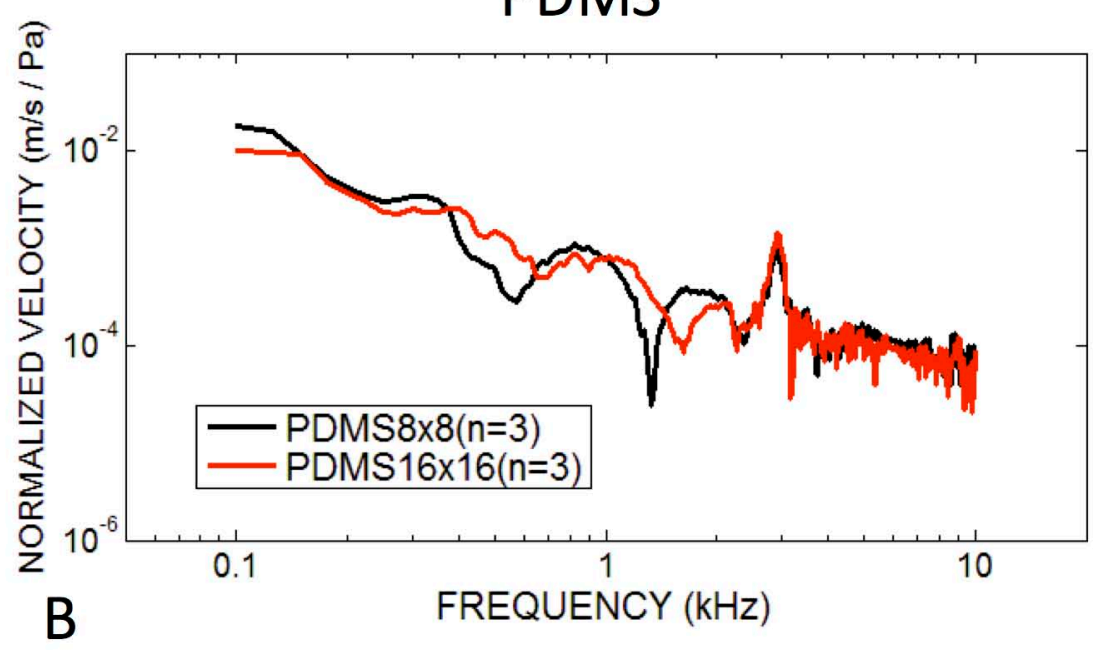

PDMS

PCL

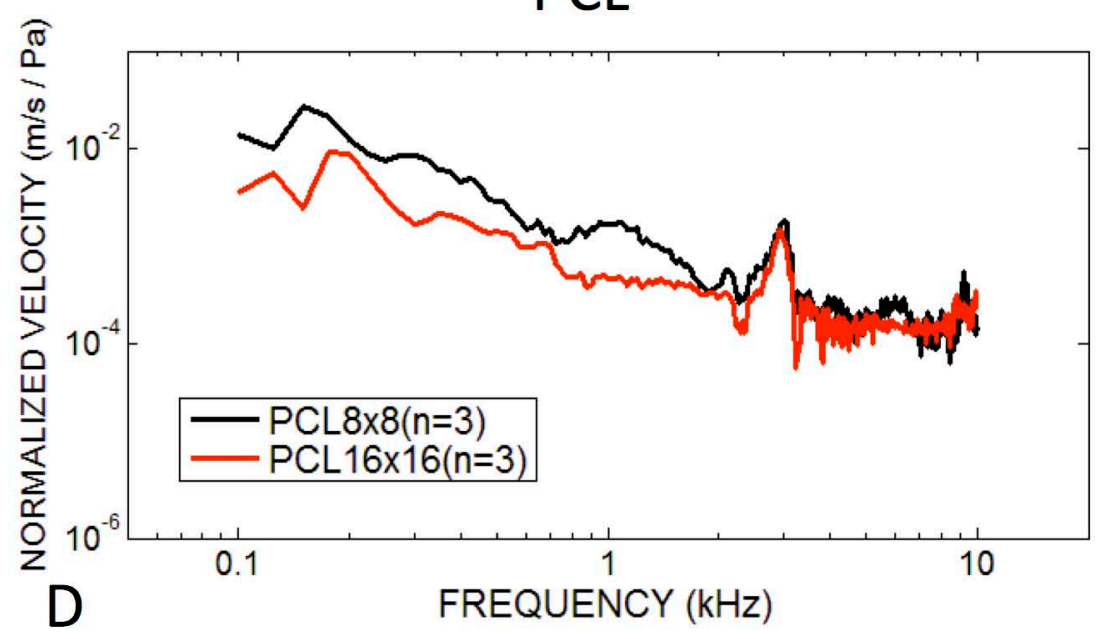




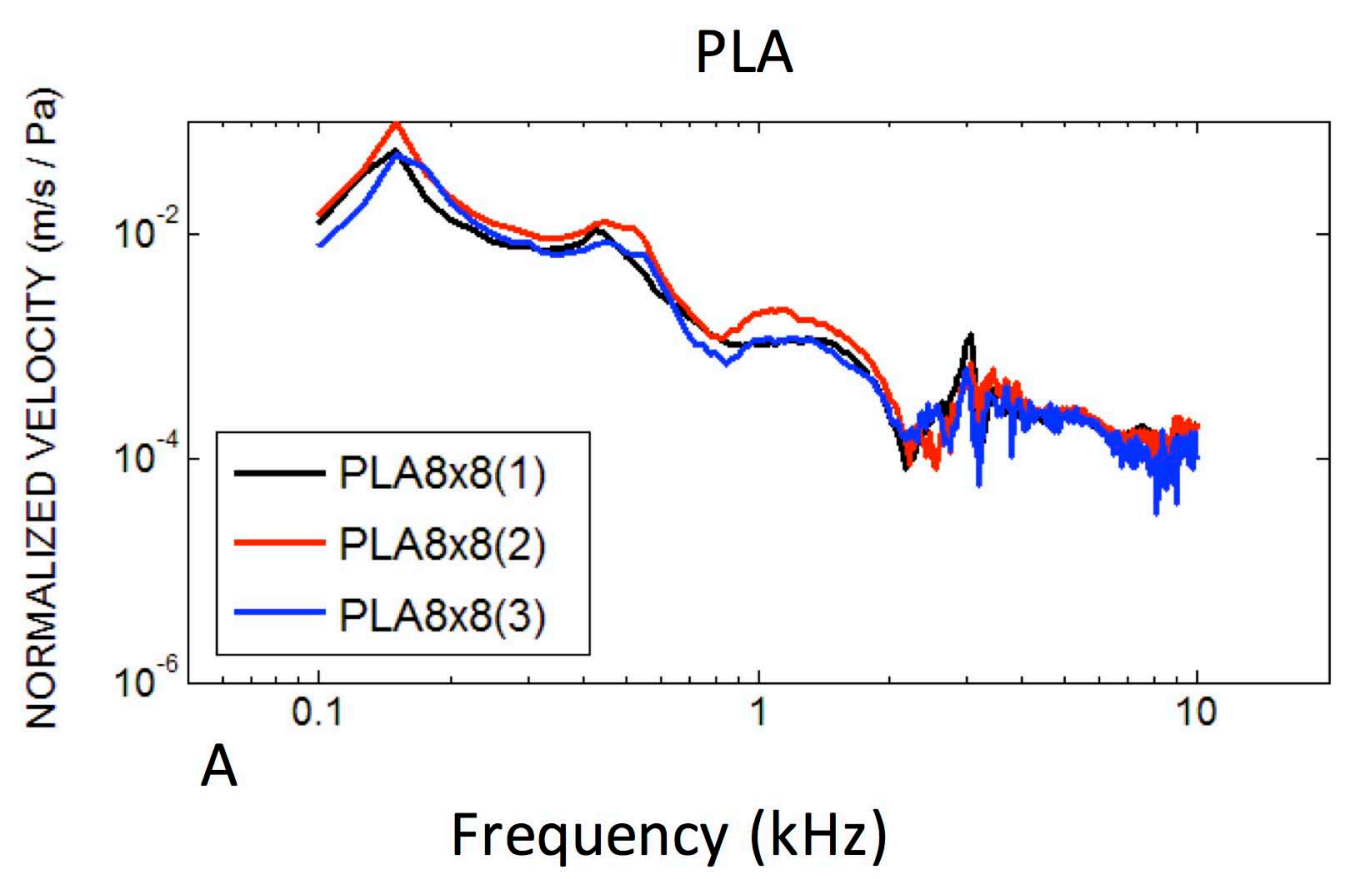

Fascia

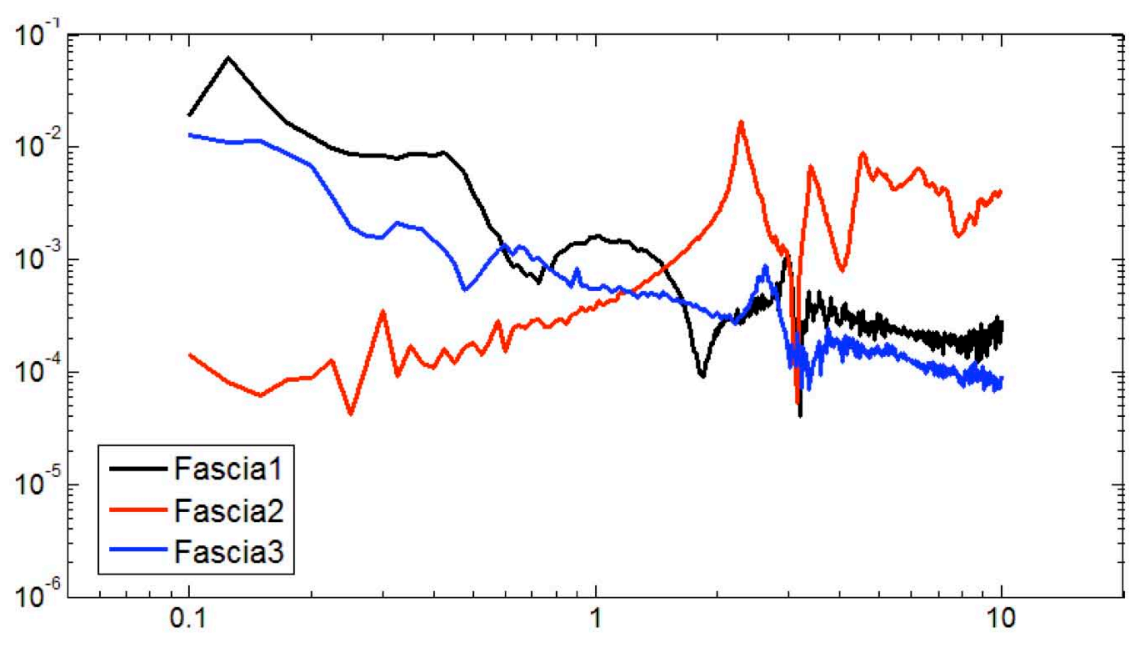

B

Frequency $(\mathrm{kHz})$ 


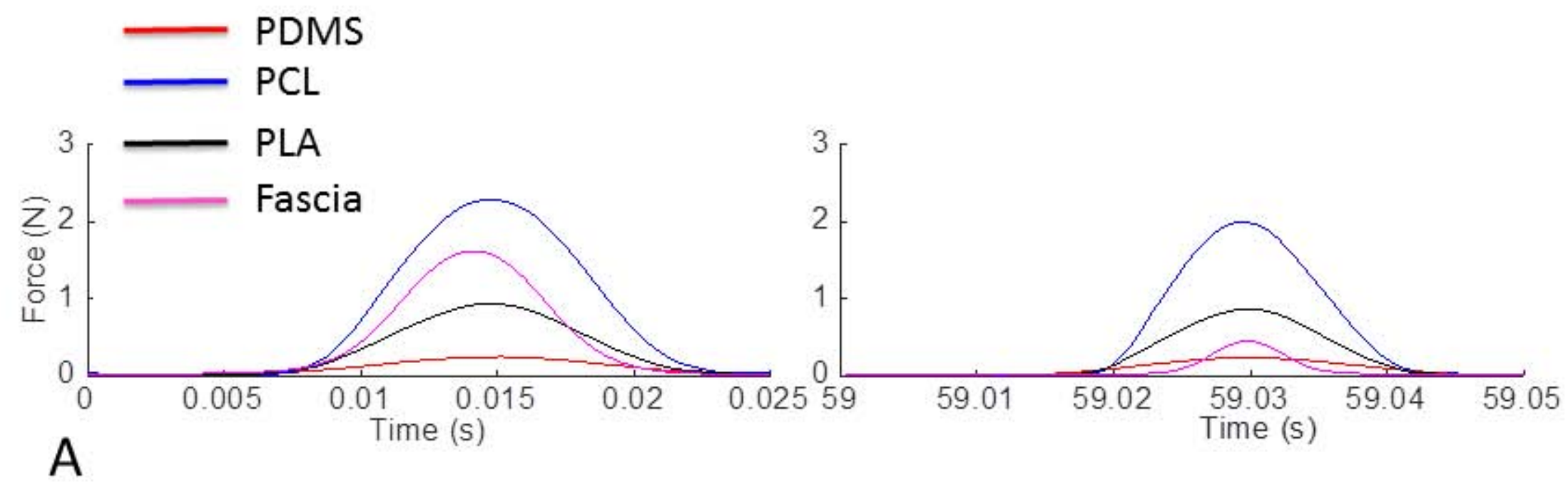



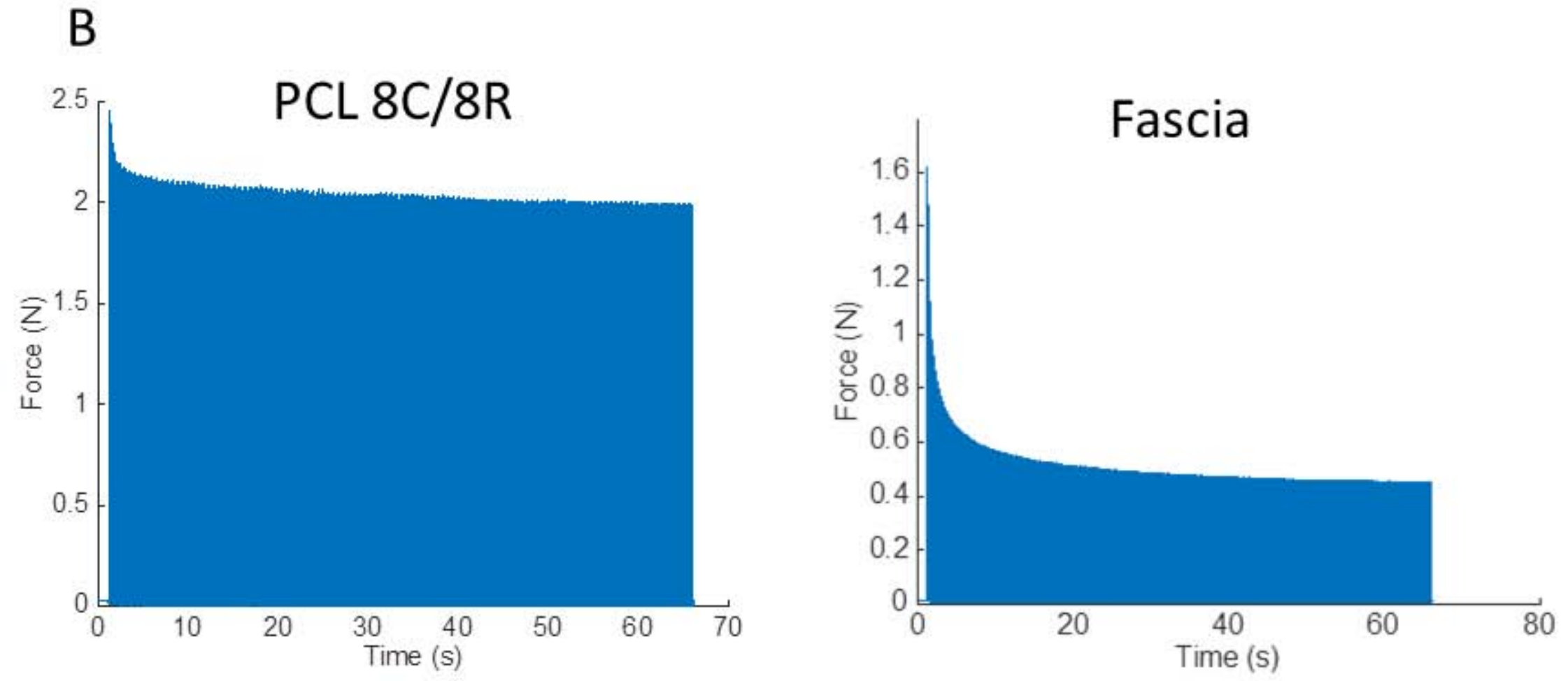


\section{PLA}

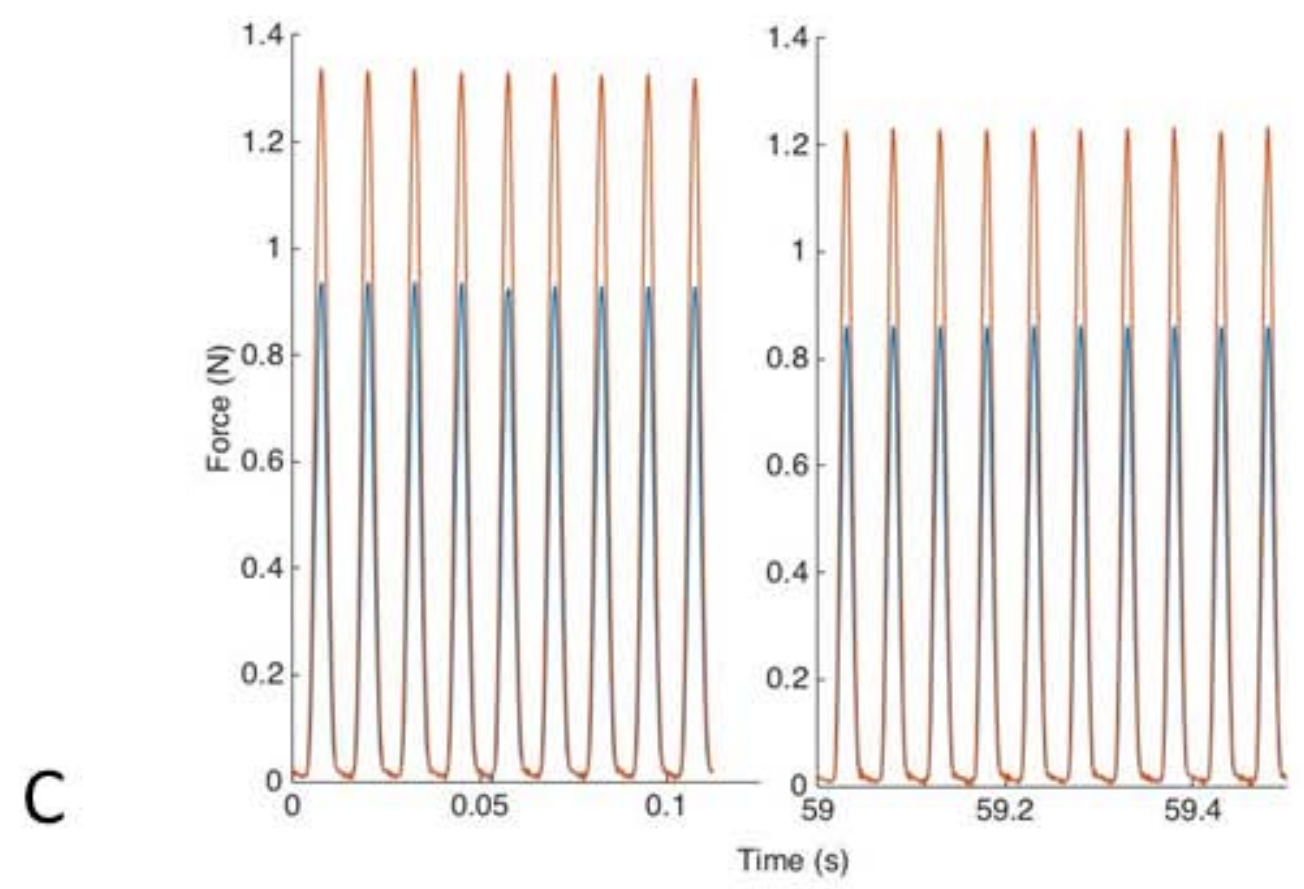

$8 \mathrm{C} / 8 \mathrm{R}$ $16 \mathrm{C} / 16 \mathrm{R}$ 